\title{
DIE GERMANISCHE CONSONANTENDEHNUNG.
}

Es fehlte bisher eine eingehende erörterung des problems der urgerm. consonantendehnung. Die entsprechende erscheinung, soweit sie ein charakteristikum der westgerm. dialekte ist, hat in der letzten zeit die fachgenossen öfters beschäftigt und bis zu einem gewissen grade ibre erledigung gefunden. Zwar bleiben auch hier noch mehrere probleme zu erledigen. Der geminierende einfluss des jod herrscht auf den westgerm. gebieten gleichmässig; aber der umfang der gleichen wirkung von $n r l m n$ steht keineswegs fest; es ist noch nicht genau ermittelt, ob sie auf alle explosivlaute einwirken und in wie weit jeder dialekt seine eigenen wege geht. Jedenfalls kann vorläufig nur die durch jod hervorgerufene consonantendehnung mit sicherheit als gemeinwestgerman. sprachcharakteristikum gelten. Wenn auf diese im verlauf auch öfters rulcksicht genommen wird, so sollen doch die urgerm. geminationserscheinungen der eigentliche inhalt der folgenden aufsätze sein, die eine vollständige vorfubrung des beurteilbaren sicheren materials und dessen erklärung bezwecken. Dabei habe ich von den gedehnten sonorlauten abgesehen, weil ich nichts neues zar aufhellung der bisher unklaren $m m$ und $r r$ zu sagen weiss. Der erste abschnitt hat mit dem eigentlichen thema nur indirekt zu tun, insofern das germ. resultat der erscheinung eine geminierte harte spirans $s$ als vertreter der idg. $t t$ ist; doch haben die theoretischen erörterungen der späteren abschnitte eine feststellung und richtige beurteilung der zuerst zu besprechenden erscheinungen zur voraussetzung; auch musste die altidg. geminata eingehender erörtert werden, ehe die entstehung von neuen, für das germ. charakteristischen geminaten gepruft werden konnte.

Beiträge zur geschichte der deutschen sprache. IX. 


\section{Indogermanische dentalgeminaten.}

Kögel hat beitr. VII, 171 ff. in einem längeren aufsatz eine theorie uber die germ. vertretung von idg. $t t$ aufgestellt, welche melurfach auklang gefunden hat. Ich hatte mir schon vor dem erscheinen jenes aufsatzes die im folgenden vorzutragende ansicht ubber das gleiche problem gebildet und dieselbe späterhin immer mehr bestïtigt gefunden, nachdem mich Kögels erörterung nicht uberzeugte. Ihr fehlen die beweiskräftigen formen, z.u iluren gunsten spricht keine isolierte erscheinung. Die s. 199 aufgeführten fälle von str (fôstr, gilstr, *blôstr) lassen eine ganz andere deutung zu: in ihnen kann str aus $s r(s s r)$ entwickelt sein (vgl. die geschichte der worte schwester, ostern): *pâttro- musste zu * fôssro- * fòsro-, * fôstro- führen. Sonst beruht germ. st gelegentlich - was Kögel s. 192 verwertet auf idg. $z d$; so habe ich auch ahd. gërsta beitr. VIII, 523 erklärt und ich kann jetzt nach einer besprechung mit prof. Hubschmann hinzufugen, dass vielleicht auch die pers. sprachen eine vorhistorische form ghrzd- zulassen. Zu den aus idg. $s+t$ suffix $\mathrm{zu}$ erklärenden germ. st gehört wol got. beist $\mathrm{cf}$. beitr. VIII, 524; ebenso got. laists (Kögel s. 188 ubersieht die idg. wz. lis, vgl. angls. leoran 'gehen' Angl. anz. V, 85); ags. fast (got. * fastus?) kann zu lat. postis gehören oder nach Prof. Hubschmann zu arm. hast 'fest'; ahd. wenist, warast vergleiche ich mit skr. vaništhu 'eingeweide'.

In andern fällen ubbersieht Kögel die möglichkeit der suffixubertragung (vgl. Kz. 23,315). So würde ich beim aufstellen eines lautgesetzes auf germ. waist, du weist' nichts bauen, weil auch aus einem lautgesetzlich entwickelten * wais (* waissa für * wóitha) auf dem wege der formassociation (gaft, maht. last, wast u. s. w.) ein waist resultieren musste; dasselbe hätte von warst (fur *wars, *warssa, * worttha) s. 199 zu gelten. Aehnlich wie sich ein suffix -stra für echtes dra-, pra- (ahd. lastar - ae. lealtor, ahd. galstar - an. galdr), wie sich ein stwa(got. waurstw) für idg. -twa- (got. piwadw, wahtwô), bes. mhd. würhte $=$ got. waurstwja) ausbildet, so konnte sich neben den alten $-t o,-t i,-t u$ in germ. ein sto, sti, stu einstellen; das prototyp des jüngeren suffixes läge in den von Kögel s. 190 angefubrten ganists, wists u. s. w.; und so ist es mir wahrschein- 
lich, dass sich auf diese weise das an. hlass zu ae. hlcest $\mathrm{n}$. 'last' verhält; so fasse ich abd. mast für *massi- als junge bildung. Uebrigens könnten auch die s. 200 angefuhrten, später erklärten bildungen wie got. ansts, alid. chunst, brunst u. s. w. dem suffix -st zu grösserem gebiet nach und nach verholfen haben.

Ich gehe nicht auf alles material Kögels ein, da er selbst nicht jedem behandelten worte beweiskraft zuerkennen wird; zudem habe ich einzelne seiner beispiele seitdem schon in meinem etym. wb. zu behandeln gehabt und dort die entwicklung derselben auch mit rücksicht auf unser problem verfolgt. Auf grund der vorigen bemerkungen, welche an Kögels hauptpunkie ankniipfen, darf ich mich dahin aussprechen, dass aus alter dentalgeminata unter allen umständen $s s$ hervorgeht, welches auch immer die stellung des accentes gewesen sein mag; jedenfalls lïsst es sich durch keine isolierten formen wahrscheinlich machen, dass der accent die idg. $t t$ zu einer doppelentwicklung $s s-s t$ gefuhrt hätte. Ich vermute dass wie $k t$, pt im germ. $\chi t, f t$ wurden, alte $t t$ zu $p t$ (dann zu $s s$ ) wurden, und diese mittelstufe möchte ich in der lat.-germ. namensform Chatti 'Hessen' annehmen.

Gegentuber dieser einheitlichen erklärung der germ. erscheinungen könnte einer geneigt sein lieber an Brugmans lösungsversuch des problems in Morph.-Unt. III, $131 \mathrm{ff}$. anzukntipfen: hier wird das problem als ein urindogerm. behandelt und zwar im anschluss an bemerkungen Krăuters und Verners, von denen der letztere die entwicklung eines reibungsgeråusches zwischem doppeltem $t$ als unwillkurliches ergebnis der artikulation ansieht. Er wie Brugman erklären also germ. wissa 'wusste' aus urgerm. * witsta, und Brugman sieht hinter diesem gresetz eine uridg. lautregel, wonach 'die affricierung dentaler explosivlaute vor $t$ schon vor der völkertrennung eingetreten sei'. Verner hatte nicht in betracht gezogen, dass die urgerm. und die idg. dentale gewiss interdental gewesen sind: also vicht * witsta, sondern * witbta müsste als urgerm. form vorausgesetzt werden; und von hier aus ist, denke ich, eine vermittlung meiner annahme mit Brugmans theorie nicht schwer. Nimmt man den von Krïuter und Verner befurworteten einschub eines reibungsgeräusches zwischen $t t$ an - dabei wird 
vorausgesetzt, dass gemeinidg. damit eine zweifach artikulierte tenuis, keine lange tenuis gemeint ist - 80 mag immerhin schon uridg. daraus $t p t$ geworden sein: das germ. musste diese lautgruppe gewiss zu $p t$ machen, solange die interdentalen bestanden. Ganz mit uurecht will Brugman dem von Müllenhoff und Kögel richtig gewürdigten Chatti das hohe alter abstreiten, wenn er schliesslich (s. 133 anm.) auch meint, möglicherweise sei germ. $s s$ eigentlich $p b$, welches in $t$ ubergegangen sei. So halte ich denn an einer germ. grundform *wipta fest und lasse dabei die möglichkeit einer idg. grdf. * witptêm (freilich nicht * witstêm) offen. Dass dies $p t$ dann durch interdentales $b p$ zu ss wurde, scheint mit durch Chatti nicht grade empfohlen zu werden; aber der von Kögel angenommene entwicklungsgang $(t t<t t<t s<s s)$ durch diese älteste form ganz zur unmöglichkeit.

Iu Kögels aufsatz vermisse ich die erörterung eines Morph. Unt. IV, 77 erwähnten punktes, die behandlung von $s s$ nach langer silbe. Allerdings wird ahd. mussa als ältere form gegen got. môsta richtig erkannt, aber eine gemeingerm. grundform mit ss vorausgesetzt (môssa s. 186). Mir scheint urgerm. kürzung von ss sicher, wie denn auch von Bahder verbalabstr. s. 65 ein germ. haisi- (ags. hôes) 'befehl' für * haissi-, haiptivoraussetzt. Hierher stelle ich auch as. ae. môs, ahd. muos für * mâtto- (môssa-, môsso-) als verwandten von got. mati- 'speise'; got. gaveisôn 'besuchen' gehört mit lat. visere zu der idg. wz. $w \breve{\imath} d$ 'sehen, wissen', zu welcher das alte to- particip wiso(Osthoff Morph.-Unt. IV, 77) 'weise' - im anord. auch nit der passivischen bedeutung des gemeingerm. gawisso- 'gewiss' bewahrt (das im got. einmal bezcugte unwisa- 'ungewiss' halte ich fur einen schreibfehler für unwissa-, vgl. got. mipwissei 'conscientia'). Hierher gehören fernerhin: ahd. iss, ags. $\hat{e} s$ 'as' zu ëzzan, wz. idg. $\vec{e} d$ (cf. lat. êsus); ahd. ôsi neben gleichbed. got. aupida 'wïste'; ae. glîsian 'glänzen' neben glitnian; anord. eisa 'gliuhende kohle' neben ae. $\hat{a} d$ 'scheiterhaufe' (gr.

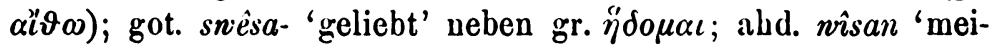
den' (altes to- praesens) zu lat. evitare; got. anabûsns, usbeisns $\mathrm{zu}$ biudan, beidan; ae. wrâsen 'kette', ahd. reisan (germ. wraisno-) zu wripan.

Scbliesslich bleibt noch eine weitere bemerkung uber idgr. 
dentalgeminata tibrig, weil sic ftir germ. $z d$ in betraclit kommt. Bartholomae hat in seinen ar. forschungen s. 24 auf yrund arischer erscheinungen die frage aufgeworfen, ob idg. $d h+t$ $(d, d h)$ als suffixanlaut bereits uridg $z u$ zdh getvorden sei? cr erkannte s. 176 auch in dieser erscheinung den letzten grund einiger germ. $z d$. Dieser annahme schliesse ich mich an, da germ. huzdo- 'hort' als altes to-particip zu ags. hŷdan 'ver-

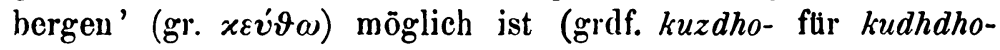
aus $k u d h-t \dot{)})$ und Benfeys erklärung von got. razda aus ir. rádim und rôdjan 'sprechen' jetzt denkbar wird (razdâ- aus *razdhâ-,

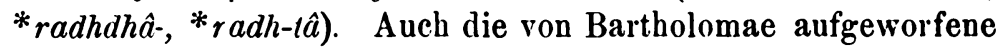
frage, ob $g h+t$-suffix uridg. zu $g d h$ geworden sei, wie das ar. vermuten lasse, glaube ich auf grund eines einzigen germ. beispieles mit ja beantworten zu müssen mit der modification, dass vielleicht ghdh statt gdh als idg. gelten muss. Zu dem nominalstamm germ. hugi- gehört das bisher unerklärte verbalabstraktum got. germ. gahugdi- (cf. hugjan, ae. hycgan); auf idg. *kukti- kann es natülich nicht beruhen, es muss auf * kughdhi- zuriickgehen und doch aller berechnung nach das suffix -ti- baben; also *kughdhi- aus *kugh $+t i$. . Freilich darf man nicht wie meist geschieht (auch bei Bartholomae s. 24) ein idg. dhugh + têr 'tochter' annelımen; denn germ. dohtêr

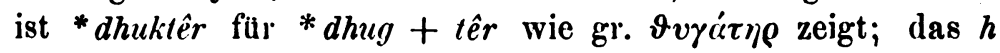
ron skr. duhitar ist europ. idg. $g$ wie in aham, hanus, mahat, hasta (áyootós): ich nehme dhugater-, dhuktr- als die idg. stammformen für tochter an. $\mathrm{Ob}$ die schw. praeteritalbildung auf - $d a$ auf - $d h \hat{e} m$ zurlickgeht, bleibt unsicher; aber man darf praeterita wie ae. hogde, sagde nach der erklärung von *gahugdiauf cin suffix $-t \hat{e} m$ lautlich wol zurückfuhren; *kugh-têm $<$ *kughdhêm < * huzðê ; *sagh + têm<*saghdhèm $<{ }^{*}$ sazð änlich kann as. habda, ags. hrefde auf*khabhdhêm für khabh + têm berulien. Wie dem aber auch sei, die nächste vorstufe für die germ. $\delta^{\dagger}$, $\delta \delta$ war immer ghdh, bhdh; aber germ. dd aus $d h d h$ sind undenkbar, weil dafiir nach einer uralten regel $z d h$ eintreten musste.

Möller hat beitr. VII, $474 \mathrm{ff}$. eine eigenartige theorie tiber

1) Der ausfall des mittleren vocals stimmt zu patér : ptros; vgl.

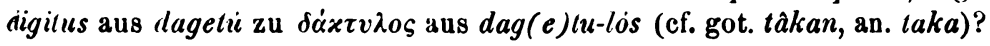


die germ. gd, bd aufgestellt: um gahugdi-, sagda, habda zu elklären nimmt er ein urgerm. synkopierungsgesetz an, wonach von zwei kurzen unbetonten silben ein mittleres $a$ vor der hochtonsilbe schwindet, wenn diese mit spirans oder verschlusslaut beginnt. Ich läugne, dass ein solches gesetz zur erklärung der praet. sagda, habda nötig ist und dass zu einem verb hugjan ein nomen * hugadi- gebildet werden konnte, wie denn auch ahd. gidult gewiss auf $t$ lit (nicht talati) beruhen muss. Die durch Bartholomaes schrift nahegelegte annahme von idg. ghdh, bhdh löst die schwierigkeit am einfachsten. Und so kann ich auch Möllers behandlung der frage des idg. $t \ell$ nicht gut heissen. Er schliesst sich an Kögel an, hält aber mit Brugman $b p$ für die vorstufe von germ. ss und Chatti fur ein *Xapbôz, wobei er unberlucksichtigt lässt, dass ein germ. $b p(=$ ahd. $l l)$ davon ebenso verschieden gewesen sein müsste wie ein $t t$ in Chatti gegen das got.-germ. $t t$ (etwa in skatto-). Auch jene von Möller gegen Kögel vorgeschlagene regel, dass idg. $t t$ nach liquiden und nasalen vor der tonsilbe $\mathrm{zu} p$ im germ. wurden, halte ich nicht für zwingend, so lange man das germ. furso- 'bereit, eilig' als to-particip zu ae. fundian 'eilen, streben' und zu ahd. fendo (aus fanpjo) 'fussgänger' zieht (grdf. $p \mathrm{n} t \boldsymbol{t}^{-}$-). Ich halte diese zusammenstellung für zweifellos und gebe daher weder Kögel noch Möller recht, zumal ahd. zinsilo 'fomes' neben zunten (suffix idg. $t$ ljo) nun begreiflich wird. Dass das aus $p$ nttó- entstandene funssó - der gleichen umwandlung $\mathrm{zu}$ funsó - erlag wie haissi (= kaitti) zu haisi(ae. $h \hat{e} s^{\prime}$ ' befehl'), ist naturlich.

Ich erkenne uberhaupt nur den wandel von $t \iota$ in $s s$ als regulär an und halte es dabei fur irrelevant, ob ein vocal oder ein sonorlaut vorhergeht und ob das dentalsuffix oder die wurzelsilbe betont war. Freilich sind keine sicheren fälle mit wurzelbetonung vorhanden, da die dentalsuffixe meist betont sind. Die kategorien, welche Kögel als stütze seiner theorie annahm, die 2. sg. praet. (waist) und die suffixform stro- (blôstr, gils(r) sind nicht beweiskräftig.

Zur erklärung jener dunklen st bei verbalstämmen mit $n n$, woruber nun oft resultatlos gehandelt ist, möchte ich von got. ansts aus einen schluss wagen. Dies ist nämlich durch seine verbreitung als das älteste beispiel fur jenes st gesichert. 
Alle iibrigen beispiele diurfen niclit das glciche alter beanspruchen. So kann got. bruns/s (in alabrunsts 'brandopfer') gegenitber dem gemeingerm. brando- (an. brandr, ae. brand, ahd. brant) gewiss nur analogiebildung nach dem oben s. 150 behandelten muster sein; oder man erkläre, warum bei dem $n n$ des verbalstammes nicht npo- oder nsto- im gemeingerm. verbalnomen erscheint. Offenbar berubt das gemeingerm.brandoauf der echien wz. bren (cf. ae. bryne, an. bruni 'brand'). kunst ist bloss hd. (dafür got. kunpi) und unterliegt wie got. ahd. brunsti- dem verdacht gleichfalls junge bildung zu sein. Kennen wir nun die wurzel zu got. ansti- (ae. êst)? Nein! aber könnte sie nicht ans gelautet haben? Das praet.-praes. ahd. unnum, ae. unnon könnte auf *unzum (: gg. ann = durrum : sg. darr fur dars) beruhen. ${ }^{1)}$ Dann könnten die 2. sg. got. anst, darst für alid. chanst, (aber got. noch kant), die praet. unsta, dorsta fur ahd. chonsta das vorbild gewesen sein. Zunächst sind ja nur die praet.-praesentia in die nst-frage verwickelt. Was weiter in betracht kommt, kann unter den hier aufgedeckten gesiclitspunkten mit demselben rechte betrachtet werden als bisher unter andern. So können wurst, schwulst entweder auf wz. wrs, $s w 1 s$ beruhen oder analogische formen nach dem s. 150 aufgestellten typus sein ; wurst zu lat. vertere 'drehen' gezogen könnte auf wursi- fur wurssi- = wrtti- berulien. Und so hat auch mhd. runst fü ahd. runs sein $t$ bezogen (cf. ne. bihest aus ae. $h \hat{e} s)$. Einer umgekehrten analogie muss das bloss nord. verbalabstrakt ofund 'missgunst' gegen ahd. abunst $=a \mathrm{e}$. cest sein dasein verdanken wegen des gemeingerm. ansti-, wozu wohl ans 'Gott' = 'Gnädiger' gehört.

Hatte man bisher fälschlich aus der natur des $n n$ (resp. $n w)$ die genesis von germ. nst begreifen wollen, so hatte man mit der entstehung von $n p$ aus $n n+d h$ oder $t$ nicht mebr gluck. Widerum ist die geminate $n n$ nicht der grund für die dunkeln $n p$. Mit der annahme eines $t \hat{e}-\left(t \hat{o}^{-}\right)$aorists

') Die angleichung von $n z$ in $n n$ kann nach den beitr. VIII, $521 \mathrm{ff}$. beigebrachten analogen erscheinungen nicht befremden: besonders ist auf den parallelen iibergang von $z n$ in $n n$ zu verweisen 8. 525. -- Im text babe ich auf folgende späte nachbildungen von anst, chunst (Haupts Zs. 21, 42i) keine riicksicht genommen: whd. begunst zu beginnen, gespunst zu spimnen, nhd. gewinst zu gewinnen. 
kommt man für das germ. vollkommen aus ohne neue lautregeln.

Dass die formen sagdế-, habdế-, libdê- der praeterita und die entsprechenden participia sagdo-, habdo-, libdo- (Beitr. VII, $144 \mathrm{ff}$.) sich mit den suffixen idg. $t \hat{e}-, t \hat{o}-$ vertragen, wurde bemerkt. Praet. wie worhtê-, puñht $\hat{e}-$, sôhtê- u. s. w.) Beitr. VII, $138 \mathrm{ff}$.) beruhen auf idg. wrktê-, $t \mathrm{n} k t \hat{e}-$, sâktê- (mit $k$ für wurzelhaftes idg. $g$ ). Ueberhaupt fugt sich die germ. aoristbildung bequem unter die annahme eines suffixes $t \hat{e}-$. Unklar ist das

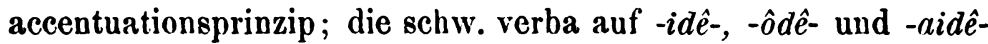
mögen oxytona oder proparoxytona in vorbistorischer zeit gewesen sein. Die primären bildungen zeigen differenzen; jedenfalls weisen auch die praeteritopraesentia nicht mit sicherheit auf ein suffix $d h \hat{e}$ - hin. Man muss allerdings einige angleichungen zwischen partic. und praet. annehmen. Das prät. kunpêweist auf $g$ ńtê- und hat mit seinem dental resp. accent das partic. kunpo- hervorgerufen: beide formen beruhen nicht auf der wz. mit $n n$, sondern auf dem alten gen-. Bisher meinte man, das praet. musse auf der wz. kann- beruhen ${ }^{1}$ ), wie man auch das verbalabstraktum aus der secundären wurzel ableitete. Wie die germ. wz. kann (ae. cennan) 'erzeugen' ihre nomina und alle primären wortformen aus der wz. ken (idg. gen) ableitet (got. kunila- als part.; vgl. nhd. kind; formen mit nst fehlen, und $n p$ von kinpo- ist génto-, nicht gennto-), so mussen wir bei einem uralten verb wie kunnan gewiss die primäre wurzelform in den ableitungen finden. $\mathrm{Ob}$ got. mahta auf idg. máktêm (w\%. mak) beruht oder für magda auf analogischem wege eingetreten ist (ksl. maga), sowie ob ahd. tohta auf dhuktê-m oder älterem dhugh-têm beruht, und ähnliche fragen werden wol unentschieden bleiben. Auch bemulhe ich mich nicht um die frago, ob einige schw. praoterita oder vielleicht einige formen der schw. praeterita suffixbetonung hatten (wilda, skulda u.s.w.) oder in wie weit analogische einwirkung wahrscheinlich ist.

Meine stellung zu den schwebenden fragen uber dentalgeminaten und den zusammenhang derselben mit der geminierten

') Doch findet sich bei Moller Beitr. VII, 462 ein leiser zweifel, ob "wir das $n n$ wie es vorliegt der zeit vor der lautverschiebung zuschreiben dürfen“. Vgl. die bemerkung über brant auf der vorigen seite. 
nasalis haben die vorstehenden erörterungen gezcigt. Ich fasse schliesslich meine ansichten in folgende punkte zusammen.

1. Eine lautgesetzliche entstehung von germ. st aus $t t$ oder nnt resp. nndh scheint mir undenkbar; wo st auftritt, beruht es auf idg. $s+t$-sulfix (resp. $z d$ ); durch analogie hat dieses $s t$ als suffixform weiteres gebiet gewonnen.

2. Lautgesetzlich kann nur ss (nach langer silbe dafur einfaches $s$ ) aus ilg. $t t$ entstehen, einerlei wie die accentstellung im worte war und ob vocal oder consonant vorausging; vor $r$ musste sich aus $s s r(s r)$ nach bekannter regel $s t r$ entwickeln; wo st fur gesetzliches ss erscheint, liegt suffixubertragung vor.

3. Ein idg. lautgesetz veranlasste den thergang von $d d h$ $(d h d h)$, auch wo $t$-suffix vorlag, in idg. $z d h$, dessen reflex germ. $z d$ ist; dagegen erlitten die idg. ghdh bhdh (gdh bdh) keine verschiebung in $h t f t$, sondern den thbrigen gesetzen conform zu $g d b d\left(z^{\zeta} \quad \delta \delta\right)$, auch wenn ein $t$-suffix vorlag.

II. Geminierte spiranten und medien.

A. Wenn ich nun von dem eben behandelten germ. ss absehe, bleiben noch die geminationen der spiranten $b f h \mathrm{zu}$ belegen: dem got. und nord. fehlen sie, dem letzteren dialekt gewiss nicht zufällig. Das ae. bewahrt altes $h h$ in ubereinstimmung mit dem deutschen, das aber den laut zusammenfallen liess mit dem aus einfachem $k$ verschobenen $h h$. Das ae. schwankt in der schreibung des eigenartigen lautes, der sonst nur noch im auslaut existierte, nachdem $h$ im anlaut zum spiritus asper geworden war; man schriels $h, h h, h c h, c h$; fur mhd. zëchen hatte die sprache teohhian, wofur Past.-Care (ed. Sweet) 391 teochian, 8. 385.387 teohchian geschrieben wird; fur pohha begegnet P.-Care 342. 343 pohcha (me. pouhe bei Stratmann); auch erscheint gelegentlich einfaches $h$, also teohian - poha (altes einfaches $h$ war inlautend zwischen vocalen verloren gegangen, und so konnte hier $h$ wie im auslaut gebraucht werden).

Folgende beispiele von $h h$ bewabrt das ae. 
teohhian $\left.{ }^{1}\right)=$ mhd. zëchen. pohha 'geldbeutel' (vgl. mndd. poche : pocke 'blatter'), - nebenform von ac. poca 'tasche', womit da die worte wahrscheinlich entlehnt sind noch got. puggs, an. posi, ae. gepose, ae. pocc 'pocke' - baier. p/oche 'pocke' zusammenhängen. - Ae. reohha-rohha, me. reihe-rouhe 'raia, rochenfisch' - ndl. roch. - Ae. seohhe 'colatorium' zu ahd. sîha. - Ae. geneahhe 'genug'. - Ae. cohhettan 'clamare', dazu ndl. kugchen - me. coughin, ne. to cough 'husten'. - Me. sighin (ae. *sihhian), ne. to sigh 'seufzen'. - Ae. wuhhung 'rabies' zu mhd. wûchzen 'brullen'. - Ae. ceahhettan = mbd. kachen, kachzen 'laut lachen, cachinari'.

Der nachweis alter $h h$ aus dem hd. ist nur möglich, wenn ein ndd. dialect dazu stinmt; vgl. die bereits erwähnten mbd. zëchen - ae. teohhian sowie mhd. kachen - ae. ceahhettan. Sonst ist hd. $h h(c h)$ in den meisten fällen das aus $k$ entstandene. ${ }^{2}$ ) Doch glaube ich mit einiger sicherheit in zwei fällen altes $h h$ noch im mhd. zu erkennen.

$\mathrm{Zu}$ ahd. sëgansa - andd. segisna 'sense' und saga, sega 'säge' sowie an. sigðr - ae. sîpe, ne. sithe (gern. wz. seg aus idg. sek, vgl. lat. secare) gehört ahd. sëh - gen. sëhhes, mhd. sëch 'pflugmesser'; hierfur kann kaum ein germ. *seka- als grundform angenommen werden; auch kann sëch ebensowenig lehnwort sein als sichel. Daher möchte ich altes hh vermuten; es besteht dann grammatischer wechsel zwischeu ahd. sëgansa und altgerm. sëhha-. Aehnlich scheint mir ahd. scahho 'promuntorium, landzunge' - mbd. schache swm. 'stlick einzelstehenden waldes' behandelt werden zu müssen wegen an.

1) Daneben muss ein westgerm. tëhon mit einfachem $h$ bestanden haben; davon kenne ich ae. praet. teode (Beow.) und part. geteod, das genau dieselbo bedeutung wie geteọhod hat; of. das glossar zu Blickl. Hom. s. 318; als inf. wäre "geteogean vorauszusetzen. Der auch von Sievers ags. gramm. $\S 403,2$ angesetzte inf. teon ist wol verkehrt. teoð Beow. 2527 steht regulär für *teọag.

2) $h h$ aus $h h j, h j$ bleibt hier aus dem spiele; iuber lahhên vgl. weiter unten. Ein weiteres beispiel für ahd. $h h$ aus $h j$ erkenne ich in abd. $i r$-bluhhen, praet. irbluhila 'flagrare, ardere', die Graff III, 240 verkennt. ferner in merihha f. 'mähre' aus marhjô- (marihjô), nom. urgerm. marhî (für mirhwî̀? eigentlich margnî? vgl. an. ylgr); hiernach sollte man auch firihha als nom. plur. zum dat. firahim lautgesetzlich erwarten, wobei die analogie des dat. wol $h h$ beseitigt haben mag. 
skógr 'hain' - skrngi 'nicdriges kleines vorgebirge' -. me. schaze - ne. shan 'dickicht', (Leo 639 gibt ae. scẹga sceacga 'buschwald' ohne beleg); dem $g$ der ausserdeutschen dialecte kann westgerm. $h h=$ ahd. $h h$ sehr gut entsprechen, während hd. $h h=$ germ. $k$ in diesem worte kaum angeht. Aehnliches hat wol auch von ahd. zuhha: zuga 'runzel' zu gelten, wofur die andern dialecte keine entsprechung haben; Graff V, 620 zieht es zu germ. wz. tuh 'ziehen'. Vielleicht stehen ae. bodig 'körper, leib' und ahd. botah (pl. botahha) 'körper, leib, leiche' im gleichen veruältnis; ähnlich alıd. botahha : botaga 'bottich, dolium, cavella'.

Ae. beispiele für ff sind: woffian 'delirare, lärmen' Leo 7, Angl. II, 528. - lyffettan 'schmeicheln'. - wlceffetêre 'narr'? Germ.XXIII, p. 403. - hoffing 'orbis'. - gaffelung 'obscenity' Hom. (Aelfr.) I, 306; II, 218. - snoffa ${ }^{1}$ ) swm. 'schnupfen'. dunkel ist mir piffe 'defruto' Haupts zs. 9, 408.

Für germ. $f f$ im hd. hat dasselbe zu gelten wie für $h h$ : bd. $f f$ enthält germ. $f f$ und germ. $p$, letzteres in den allermeisten fällen. Man kann aber die frage aufwerfen, ob altes $f f$ im hd. nicht zu $p f$ geworden ist. Wie will man anders hd. opfarôn (ae. offrian) aus offerre erklären? doch nicht aus obferre? Isidor hat hepfan für got. hafjan. kripfa wäre aus krifjô besser zu begreifen als aus *kripjô (Holtzmann ad. Gr. 309), weil dann grammatischer wechsel mit as. cribbia besteht. Auch könnte ahd. chapfên als intens. $p f=f f$ haben. Im ahd. besteht neben hevo-heffo 'hefe' hepfo, mhd. nhd. (Winteler) hepfe neben heve, heffe. Anderseits spricht ahd. laffa 'band' (got. lôfa) für ff als hd. vertreter von altem $f f$, desgleichen die mhd. doppelform schroffe : schrove fwm. 'felsklippe, steinwand', deren reflexe noch nhd. dialecte bewahren, s. Schade.

B. Das material die schicksale des alten $p p$ zu verfolgen ist leider sehr klein. Das hd. $t t$, welches aus $p p$ entstehen muss, ist allein nicht beweisend, weil ihm auch $d d$ zu grunde

1) Vgl. ndd. snüffeln bei Jellinghaus Ravensb. Mundart p. 51; die ebendaselbst angeführte gaffel (ebenso ndl.) 'gabel', naffel 'nabel' (ndl. navel), duffert 'tauber' (ndl. doffer), schüffel (ndl. schoffel) 'schaufel' werden wol nicht auf urgerm. consonantendehnung weisen; auch drüffel 'schar' (zu ahd. druba 'turba minor') gehört hierher. 
liegen kann; erst die ibercinstimmung des engl. $b$, i) nit hd. $\iota$ spricht fur altes $b b$. Die wenigen sicheren beispiele, welche sich uns bieten, machen für das nord. und ndd. eine verschiebung von $b p \mathrm{zu} t t$ wahrscheinlich ${ }^{2}$ ); so kann es dann kommen dass hd. und ndd.-nord. scheinbar gleiche dentalstufe zeigen.

ae. moppe swf. - ne. moth 'motte' - mbd. motte, dagegen an. motti nom., ndl. mot. In demselben verhältniss steht ahd. spottôn zu an. spotta, ndd. ndl. spotten; leider feblt ae. * spoppian - me. *spopbin; doch setzt ahd. spottôn ein *spoppôn voraus, da aus einem * spoddôn das ndd. spotton - an. spotta nicht zu erklären ist. Für das nord. sind dies soviel ich sehe die beiden einzigen beweiskräftigen formen. Für das ndd. kommt noch hinzu ahd. latta - me. lapbe, ne lath, aber ndl. lat, ndd. latte. Bei dem worte ratte liegen allerhand bedenken vor; hd. ratta aus rapba stimmt nach dem aufgestellten gesetz zu mndl. rotte - nndl. rot; an. rotta ist nach Vígfúss. s. v. entlehnt, und auch ins me. ne. (me. ratte, rotle, ne. rat) scheint das wort aus dem ndd. ndl. entlehnt zu sein. Vielleicht haben wir es hier mit einem gemeingerm. lehnwort zu thuen, dann verlöre es hier seine beweiskraft.

Fưr das ndl. scheint das angeführte gesetz eine einschränkung zu bedtirfen. Wir finden nämlich für altes $p b j$ statt $t \ell$ vielmebr ss wie bereits J. Grimm zu Reinb. 224 erkannte: ndl. wisse $=$ mbd. witte, ae. wippe (cynewippe); smisse $=$ ahd. smitta aus smibja. Hierher gehört vielleicht auch ndl. klis 'klette' = ahd. chlëtıo, ae. clipe; auch bei diesem worte, dessen formenreichtum das $\mathrm{DWb}$. vorfubrt, bleiben allerlei zweifel. Weist mndl. pitte 'mark' - nndl. pit gegenluber ae. pipa ${ }^{3}$ ) ne. pith auf eine ae. nebenform * pippa?

1) Weitere beispiele fur ae. $p$, sind $z u$ den im text behandelten die bei Holtzmann ad. Gr. s. 216 verzeichneten worte; davon verhält sich ae. rypla (hryppa?) zu ahd. rudo, mhd. nhd. rüde, (aber hers. rülte) wie an. voopvi zu ahd. wado 'wade', ahd. grâvio : ahd. grâvo; vgl. Pauls instructiven aufsatz Beitr. $7,113$.

2) Vielleicht galt für das got. das gleiche gesetz: falls got. alta 'vater' und hd. atte 'grossvater' identisch sind, muss des hd. wegen $p p$ zu grunde liegen; dann wäre Ezzilo Etzel die lautverschobene got. namensform Attila, denn die echte hd. form müsste Elttilo sein. Doch ist über die ganze sippe nicht ins reine zu kommen.

3) Vgl. mndd. pedek, peddek 'mark' bei Schiller-Lübben III, 312. 
Ueber die vertretung von germ. $h$ h im nord. lässt sich kaum etwas probables vorbringen. $\mathrm{Ob}$ an. $t e ́$ in der phrase láta $i$ té als 'anordnung, bestimmung' zu fassen ist und zu mhd. zëche - ae. teoh $(h=h h)$ gehört wie Schade will, bleibt zweifelhaft. Sicherer ist die zusammenstellung von ae. reohharohha 'raia' mit dän. rokke - schwed. rocka 'raia': dann wäre $h h \mathrm{zu} k k$ geworden wie $b p \mathrm{zu} t t$. Im ndl. heisst der rochenfisch roch; dem ndd. ist der laut hh nicht fremd, obwol es $p b$ zu gunsten von $t t$ aufgegeben hat vgl. ndd. petten 'gehen' - mbd. phetten (:pfeden) 'gehen' = ae. pappan.

C. Während für die in den späteren sprachperioden erscheinenden medien für die ältere zeit noch weiche reibelaute anzunehmen sind, herrscht in der dehnung die media, vgl. an. $d d$ neben $\delta$, an ae. as. $b b$ gegen $f(\hbar)$, ae. $c g$ gegen $g$. Dem got. fehlt gedehnte media wie gedehnte tenuis; von dem zweideutigen $g g$ muss gänzlich abgesehen werden. Das gebiet der $d d g g b b$ sind die westgerm. dialecte und das nord., welches letztere ubrigens mit dem got. den mangel gedehnter harter reibelaute teilt. Die aufgabe dieses abschnitts ist es, nicht umlautende geminationen der mediae in diesen dialecten nachzuweisen und die häufigkeit ihres vorkommens damit zu charakterisieren.

Ae. frocga swm. 'frosch' - ne. trog. - Ae. docga m.? in den Prudentiusgl. Germ. 23, 399 'hund' - ne. dog. - Fur ae. focge swf. 'fuchsin' bei Leo fehlt mir ein beleg1) (vgl.got. faủhô). - Ae. flocgian 'emicare' (Germ. ibid. 399) - mhd. flocken swv. 'fliegen'. - Ae. raggig (zu an. rögg 'villus') 'setosus, borstig' gehört vielleicht nicht hierher. - Ae. clucge (= ahd. glocka) wiegt als wahrscheinliches lehnwort nicht schwer. - Ae. hocgian 'eminere' Germ. 23, 392.

Ae. scẹabb (pl. scẹabbas P.-Care s. 64) 'scabies'. - gabbian 'deridere', gabbung 'derisio', entsprechend an. gabba swv., gabb

1) Focgan- als erstes compositionsglied in ortsnanen belegt Kemble (od. Dipl. Wenn ich in die obigen sammlungen die ungeheure fülle von ahd. und ae. eigennamen aufnehmen wollte, was bei der unsicherheit und dunkelteeit ihres ursprungs meist wertlos wäre, so künnte das geminationsmaterial bedeutend vermehrt werden. 
n. - Ae. ëbba swm. 'ebbe' zu got. ibuks. - Ae. crabba, an. krabbi swm. 'krabbe'. - lobbe f. 'aranea'. - Ae. scrobb 'staude, gesträuch' - ne. shrub. - Ae. drabbe (Leo) swf. 'befe' = ndl. drab, drabbe 'hefe' $\%$ ahd. trab - ae. draf 'hefe'. - Ae. *sobbian swv., me. sobbin, ne. to sob 'seufzen' (mit ae. seofian zu ahd. sûftôn); me. snobbin 'seufzen'.

Ae. budda 'käfer' (Aelfr.-Glossar). - poddettan 'pulsare Germ. 23, s. 399. - broddian (neben brottettan) 'luxuriare, infructicare' Haupts zs. 9, 435. - Ae. me. rudduc 'rotkelchen'. - Ae. sceadd - ne. shad 'maifisch' (Leo). - Ae. codd, an. koddi 'tasche'.

Deutsche belege (auf beispiele für deutsche $t \iota$ wurde vezichtet, weil sie auch aus $p p$ entstanden sein können).

Ahd. waggo (* wacko) - mhd. wacke swm. 'eine art steine'. - Abd. rocko, as. roggo nom. 'roggen'. - Ahd. glocka 'glocke'.

Abd. lappa 'lappen' aus * labba (aber dafür ae. lappa). Ahd. trappa 'tenda' aus trabbôn (aber dafür ae. trappe). Ahd. chluppa, mhd. nbd. kluppe zu wz. klüt. - Ahd. happa, hâppa 'hippe, sichel', vgl. pfälz. hoob 'sichel'. - Mhd. nhd. krüppel (nach dem Dwb. nicht aus dem ndd. entlehnt) aus krubbil (aber dafür ae. me. crypel - ne. cripple, an. krypill). - mhd. knappe aus * knabbo; mhd. rappe, ahd. rappo (schweiz. rap bei Winteler s. 50) 'rabe'. - Ahd. trappo (trâppo?), mhd. trappe masc. 'traubenkamm, racemus'. - Ahd. ippihhôn 'revolvere' zu ae. ëbba (Graff I, 92). giscoppôt (Gl. Ker.) schoppen Weinhold alem. Gr. s. 117 und Lexer. ${ }^{1}$ ) - Mhd. wacken, intens. zu bewegen, aus waggôn stimmt zu me. waggin aus ae. *wacgian.

III. Geminierte tenues in intensiven und schw. nominibus.

A. Ein reiches gebiet nimmt die geminata des stammauslauts als charakteristikum primärer und secundären intensivbildung ein. Wir unterscheiden dabei 1) schw. verba auf -ôn. 2) schw. verba auf -jan. 3) schw. verba auf -atjan.

1) Schweiz. šope (aus ahd. skoppôn) bei Winteler s. 59.

2) Eigennamen: ahd. Appo . Boppo, Noppo; Patto, Watto; Petto, Alto, Hallo (in den letzten beiden worten aus $p p$ wegen der schreibung dth, thd?); Wippo. Woppo, Noppo nach Weinholds grammatiken. 
1) Ahd. zocchôn, mhd. zocken 'zerren' zu germ. tiuhan, idg. wz. duk. - Ahd. chlocchôn (neben chlohhôn) 'klopfen', mbd. klocken; ae. cloccian 'to cluck'. - Ahd. locchôn, ac. loccian 'Incken'. - Ahd. tocchôn 'sich mühen'. - Abd. brocchòn, mhd. brocken 'bröckeln'. Weiteres bei Gerland 'Intensiva und Iterativa'.

Ae. twiccian, ahd. zwëcchôn 'carpere, vellere' neben ahd. zwigòn. - Ae. liccian, ahd. lëcchôn 'lecken' zu got. biluigôn, idg. w7. ligh.

Ae. paccian 'leniter palpare'. - Nbd. kracken neben krachen s. Dwb. - Ae. taccian, part. gelaccod 'edomitus' (Germ. 23, 402). - Ae. pluccian 'pflücken'.

Ae. hoppian, mbd. hopfen 'hüpfen'. 1) - Ae. forstoppian, ahd. stopfôn (und stoffôn) 'stopfen'. - Ahd. chlopfôn (neben chloffôn), s. Dwl. s. klopfen. - Ahd. topfôn 'punktieren'.

Ae. hnappian 'dormitare'. - Ahd. chlapfôn (chlaffòn) 'schwätzen', ae. clappian 'pulsarc' s. Dwb. s. klaffer. - Ahd. stapfôn, mhd. stapfen.

Ahd. chrazzôn 'kratzen'. - Ahd. chazzôn 'quälen'. Ae. hoettian 'schinden'. - Mhd. strolzen 'strotzen'. - An. glotta 'to grin'.

2) Mhd. hüpfen. - Mhd. lücken 'locken'. - Mhd. rüpfen 'rupfen' zu raufen. - Mhd. bücken zu biegen. - Ahd. rucchen 'rucken'. - Ahd. drucchen, ae. pryccan 'drücken' zu an. pruga 'drïcken' sowie zu ahd. drîh 'fessel' (zu der idg. wz. truk gehört, durch die nasalierte wz. trunk vermittelt, auch germ. priñhan 'dringen'). - Ahd. zucchen 'zücken' neben zocchôn. - Ahd. iturucchen (ae. eodorcan) 'ruminare'. - Ahd. smucchen, mhd. smücken zu smiegen. - Ahd. *snizzen (aus snizzari zu folgern) zu snîdan. - Ahd. nicchen zu nîgan (wz. hnĭgn). Ahd. tupfen $=$ topfôn. - Ahd. slupfen, mhd. slüpfen 'schlüpfen' (slopfezzâri). - Ahd. slipfen (part. gislipfit) 'gleiten' zu slifun (vgl. auch mhd. sleifen und sleipfen, sleife und sleipfe). mhd. schüpfen, schupfen zu schicben. - chripfen 'wissen'. mud. pflücken.

Hierher stelle ich noch zwei intensiva auf ai- : ahd. chapfên

1) Auf cine gorm. nelienform *hobbôn weist haier. hoppen (Weinhold s. 12i) unıl schweic. hopen (Winteler S. 59); vgl. ksl. kypěti. 
(neben chaffên) 'schauen'. - Ahd. bichlëpfên 'umschliessen' (ags. clyppan 'umarmen'). - Ahd. stëcchên 'fixum esse'.

3) Hieran schliessen sich die intensiva und iterativa mit dem secundärsuffix got. -atjan, woneben eine form -atjôn (und itôn?) vorausgesetzt werden muss. Ich setze bei den ae. verba uberall -ettan (nicht das auch bezeugte etan), bei den ahd. verben ezzen (nicht das auch bezeugte ezzen Holtzmann ad. Gr. s. 298) als normalform des infinitivs an, ohne rücksicht darauf, ob vielleicht nur die form -atjôn in dem einen oder andern falle nachweisbar ist. Für unsere zwecke ist die differenz atjan: atjôn von keinem belang. Ich fuhre hier zugleich mit den geminierten tenues auch medien und spiranten vor.

Beispiele für geminationsformen:

a) aus dem ae:

cohhettan 'husten, kichern'

gaffettan 'höhnen'

lyffeltan 'schmeicheln' s. Leo.

doppettan 'tauchen'

poddettan 'pulsare'

sceottettan 'saltare'

b) aus dem ahd.:

chahhezzen (ae. ceahhetian)

chrockezzen 'crocitare'

muckezzen 'mutire'

flocchezzen 'colludere'

(Holtzmann ad Gr. s. 274)

uhhizzen 'klagen' (?)

dahhezzen 'flimmern' (?)

ceahhettan 'Iachen'

vloeffettan nach nloeffetêr ‘' 'vilium

[bavilorum' s. 159 .

cloppettan 'to have beatings, to pahlocceltan 'singultare' [pitate'

brottettan neben broddettan 'fruti[care's. 162.

blëcchezzen 'blitzen'

gackezzen 'mutire'

gickezzen 'mutire'

slopfezzen 'vagari'

(slopfezzari 'circumcellio')

snëpfezzen 'schluchzen'

grockezzen 'crocitare'.

Widerum begegnen doppelformen mit und ohne geminata des stammauslauts:

ahd. napfezzen : naffezzen 'dormitare', vgl. ae. hnappian.

ahd. ropfezzen : roffezzen 'eructare'

ahd. snopfezzen : snoffezzen

ahd. tropfezzen : troffezzen, ae. dropettan

ae. siccettan : sicettan 'seufzen, schluchzen'

ae. Joccettan : rocettan 'eructare'

ae. gaffettan : gafettan

ae. liccettan : licettan

Ohne mich umzusehen wie weit die angefubrten verba in unsern ae. wwbb. belegt sind, gebe ich hier fur die selteneren einige belege aus der prosa ohne dabei dem reicheren ma- 
terial eines zuklinftigen wbs. des ac. vorgreifen $z u$ wollen. gaffettan vgl. gaffetung Hom. I, 306; II, 218, daneben gafetung Hom. I, 330 (dazu noch ae. gabbian). - * wlaeffetan nach wlaffetêre Germ. 23, 403. - cloepyettan Ld. III, 92. - boddettan Germ. 23, 399. - brottettan Haupts 7s. 9, 435. - Ae. siccettan Part.-Care s. 64, Hom. II, 120, Mone QF. 417 (an letzten beiden stellen siccetung), dagegen siccettun Past.-Care s. 65, wozu sicetung Hom. I, 614. Für das ahd. vgl. Graff. ${ }^{1)}$ Aus dem mhd. vgl. noch snupfezen, snüpfezen 'schluchzen'.

B. Unter den nominibus zeichnen sich die schw. declinationsclassen aus durch häufiges auftreten von consonantendehnung im stammauslaut.

a) Geminierte tenues: ahd. floccho 'lanugo'. - Ae. scucca, sceocca 'teufel'. - Ahd. broccho, mhd. nhd. brocke (got. gabruka stf.) zu wz. brek. - hopfo 'hopfen'. - Ae. doppa 'mergus'. - stoppa 'poculum'. - sceoppa 'gotteskasten'. - ahd. toccha 'puppe'. - stopfa 'punkt' (auch stopfo). - ae. loppe 'floh'. glappe 'klette'. - Ae. wicca, wicce (ne. witch) 'zauberer, zauberin' zu wîglêre 'zauberer'. An. tappi, ahd. zapfo 'zapfen'.

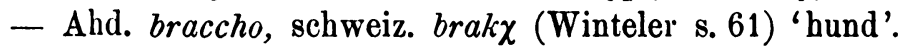

In mehreren beispielen besteht neben dem schwachen stamm eine kurzere stammform; vgl. an. bokki, ae. bucca 'bock' : an. bukkr, bokkr, ahd. mhd. bock. - Ahd. roccho 'colus' : an. rokkr. - Ahd. smoccho 'unterkleid' : ae. smocc, an. smokkr. - Ae. cnotta : an. knútr 'knoten'. - Ahd. flëccho 'fleck' : an. flekkr. - Ahd. chozzo : choz 'kleid'. - An. hnukki (ae. hnecca mit altem $\ddot{e}$; umlaut ist unmöglich weil das engl. neck dann mit palatal tch im auslaut trscheinen müsste) : ahd. nacch-. Ahd. lopfo : topf (und tof) 'kreisel'. - stopfo 'punctus': stupf.

Andere worte mit geminata im wurzelauslaut haben neben der tenuis auch geminierte media oder spirans; vgl. ae. tappa, an. lappi : ahd. lappa (aus *labbôn-). - Ae. trceppe 'falle,

1) Unberïcksichtigt sind im text einige unerklärte ae. geminaten ohne voraufgehenden umlaut im verbum (nicht speciell in intensiven). hubai- wird habban, nakai- wird *woecan (part. woecende), libai- wird libban, lakai- wird laccan, papai- wird poppan, stapai- wird stoppan. Der mangel des umlauts kann nicht durch secundïre einwirkung bestehen, muss vielmehr alt sein. 
schlinge' : ahd. trappa (aus trabbôn-). - Ndd. kluppe : ahd. chluppa (für bb) s. Dwb. s. kluppe. - Schweiz. bake (aus * baggo? s. 167) neben ahd. baccho (aus * bakko)?

Nicht selten ist wechsel von geminata mit einfacher consonanz. Ahd. stapfo : staffo (hevistapfo: hevistaffo). - Alud. tropfo: ahd. troffo, ae. dropa. - Mhd. schapfe : schaffe (ahd. scaffo) 'schöpfgefäss'. - Ahd. chinnibaccho : chinnibahho 'kinnbacken' (mhd. backe : bache). - Ahd. wituhopfa : wituhoffa 'wiedehopf'. - Ae. cnotta : ahd. chnodo, chnoto 'knoten'. Ahd. stëccho : stëhho 'stecken, stock', noch mbd. stëcke : stëche $(\ddot{e}$ scheint mir sicher wegen ae. sticca, ne. stick, weil $k j$ im ae. $c^{\prime} c^{\prime}=$ ne. $t c h$ ergeben haben müsste; also urgerm. stikkon- : stikon-). - Ahd. gapfa: gaffa schwstf. 'mitia'. Ahd. chipfa : chiffa schwstf. 'humerulus'. - laccha: lahha (st. schw. f.?) 'lache', wofur noch baier. Lacken Weinhold 187. Ahd. trûha : truccha swf. 'lade, kiste' beruht auf germ. brûh(cf. ae. prûh cons.-st., an. prỏ) : prukk-ôn, dessen geminata durch mlat. trucca und schweiz. trukxe 'truhe' Winteler s. 61 erwiesen wird. - Mhd. zëcke : zëche swm. 'holzbock' (schweiz. ze $\chi \chi$ bei Winteler) aus tikon-: tikkon- (engl. tick, tike). - Nhd. sprosse : sprotze Scherer Anz. III, $63(t: t t)$.

b) Gleiche erscheinungen zeigen sich - nur in beschränkterem umfange - bei der geminierten media; ich stelle die beispiele für geminierte spirans hinzu, zumal ahd. $t t$ sowol auf $d d$ als auch auf $t t$ beruhen kann.

Beispiele fur schwach flectierende stämme:

Ae. docga 'hund'. - Ahd. *wacko (waggo), mhd. wacke 'eine steinart'. - An. baggi (pakki) 'last'. - Ae. budda 'käfer'. - Ahd. chratto, mhd. nhd. kratte 'korb'. - An. toddi, ahd. zotto 'a tod of wool'. - Ahd. tutto 'mamma'. - Ahd. gilır̈llo, gibrëtta (neben brët, dat. sg. brëtta) 'brett'.

Wechsel einer kürzeren stammform mit einem $n$-stamme: An. koddi 'pillow' : ae. codd 'sack'.

Wechsel von geminata mit einfacher consonanz:

Abd. chnappo, mhd. knappe : chnabo, ags. cnafa (und cnapa). - Ahd. rappo (aus rabbo), mhd. rappe (s. auch Lexer unter nahtrappe, rappengesanc, rappennest) : ahd. rabo, mhd. nhd. rale. - An. vagga 'wiege' : ahd. waga, wiga (Möller Kuhns zs. 24, 507). - Ahd. truppo: trûbo 'traube' (Graff V, 252). - Abd. chlëtıo 
(aus klippo) : ac. clipa 'klette'. - Ae. frocga, ne. frog 'frosch' (: ae. frocca? doch ist dies bezeugt?). - Abd. ritto : rito 'fiber' Ahd. ratto : ahd. rato, rado (Jänicke Zachers zs. 4, 31).

Ahd. scahho : ae. sceaga, sceacga of. s. 158. - Mhd. schroffe : schrove m. 'klippe'. - Schwei\%. bake 'backe' (Winteler s. 61) aus * baggo? neben hd. baccho (chinni-)?

Die wurzel erscheint ausserdem in zahlreichen fällen mit einfacher consonanz im auslaut verwanter worte: As. roggo, ahd. rocko 'roggen' neben engl. nord. rŭgi- (s. Kuhns zs. XXVI, 101). - angls. ëbba 'ebbe' neben got. ibuks 'zurück'. - An. krabbi, ae. crabba neben ahd. chrebiz.

Gegeniiber diesen sammlungen, welche das hauptgebiet der geminaten aufdecken, ist geminata in wurzelauslaut anderer nominalstämme selten.

$o$-stämme: ahd. scopf (und scof, ae. sceop) 'dichter'. Ahd. chopfa : choffa 'crateras'. - Ahd. chropf und chrof 'kropf' s. Dwb. unter kropfbein - Ahd. chnopf und chnof (s. Dwb.; vgl. part. gichnuffit zu chnupfen). -- Ahd. scopf (neben scoff) 'wetterdach'. - Ahd. roz. - Abd. smacch- 'sapor' zu gismahhên (smecchen) 'sapere' (ae. smacc, smacian). - Ae. facg 'platesia'; puddas (Germ. 23, 399) 'furche'; gnw It 'schnake'. - An. stokkr, ahd. mhd. stock (und stoch) 'stock'. - Ahd. chapf 'cacumen'.

$i$-stämme: an. bekkr, ae. becc (aus bakki-z) m. 'bach' gegen hd. bah (aus baki-z) ${ }^{1}$ ). - An. huppr gegen ae. hype, got. hupi- (doch lïsst das an. wort noch andere erklärung zu). Ahd. rucch- 'rauch' (rukki-) neben rouh (rauki-). - Ahd. bizuch'anzug, kleidung' zu ziohan.

$u$-stämme: an. hottr, ae. heett (got. *hattus) m. 'hut' neben ahd. huot, ae. hôd.

Secundäre bildungen: zu ahd. ziga gehört das dimin. zicchîn, zicchi n. 'zicklein', zu an. kiß, ahd. chizzîn, chizzi (got. "tigô-, *kidja-:*likkein, *kittein). Hierher auch nach Paul Beitr. 7, 133 nhd. ricke (rikkiô?) zu rêh 'reh' (raiho-); vgl.

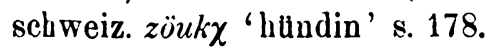

1) bakki-: baki- = skoppo- : skopo- $=a l l o-: a l o-($ got. ala-) $=$ stammo-: stamo- (Holtzmann ad. (Gr. s. 315). 
IV. Ursprung der gemination und chronologie der verschiebung.

A. Die ausbildung der geminationen $k k \| p p$ ist neben der laut- und accentverschiebung zweifellos eines der hauptmomente in der ausbildung der specifisch germ. lautform. Der idg. sprachzeit waren die geminaten durchaus fremd bis auf" $t \ell$, das aber in der ïltesten zeit des germ. den regeln gemäss zu $b t$ (weiterhin zu $s s$ ) verschoben wurde. Das jtingere germ. It findet sich in keiner verwanten sprache wider: skatto-

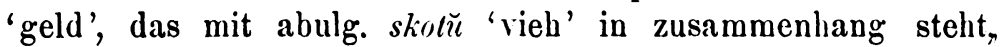
zeigt nur im germ. dentalgeminata. Für geminirtes $k$ könnte jemand auf grund von ags. bucca, an. bukkr 'bock' neben skr. bukka 'bock' eine vorhistorische geminata behaupten. Doch muss dies beispiel abgewiesen werden; denn erstens ist das ind. wort in der ganzen litteratur nicht belegt, und zweitens müsste es auf grund des germ. wortes mit media aspirata anlauten und nicht mit media. Das germ. wort bukka-n-beruht vielmehr mit dem zd. bûza 'bock' oder klarer (nach Prof. Hubschmann) mit gleichbed. armen. buts auf einem vorgerm. bhug'o (nicht bhug'ho), bhug'on-.

Ich habe in der tat kein sicheres beispiel von übereinstimmung einer germ. gemination mit einer aussergerm., uud darum trage ich kein bedenken, die ausbildung der oben behandelten geminaten $k k t t p p$ der germ. ursprache zuzuschreiben. Aber wie entsprangen sie?

Man hat ein $n$-suffix im verdacht, in der gemination $z u$ grunde gegangen zu sein; einmal weil $n$ auch noch in der gemivata $l l$ für $I n$ steckt (vgl. fulla-für plna-, fallan für phl-na-, wulló- fur $n \ln h \hat{a}$, wëllô- fur welna- u. s. w.), vielleicht auch in $m m$ (germ. swimman für *swim-nan? vgl. got. brin-nan aus wz. bren); dann auch weil kein anderer consonant existiert, der in der urgerm. zeit sonst hätte geminieren können. Dazu komnt das verhältnismässig seltene auftreten von $n$ nach explosivlauten. Von den etymologisch durchsichtigen beispielen für $n$ in verbindung mit alten tenues - ich beschrïnke mieh dabei auf das klare material, welches sich aus Fick ergibt - ergeben sich vier sichere fälle mit urgerm. wurzelbetonung: got. auhns 'ofen', rahnjan 'rechnen', ahd. lêhan (aus * laihwnaz?) 'Ichen', 
rahimnen 'rauben' (rahnjan). Ausserdem ist aus griinden des vocalismus wurzelbetonung warscleinlich in got. airkns 'gut', swikns 'rein', rign 'regen' und ags. swefn 'schlaf'. Jedesfalls findet sich kein unzweifelhaftes germ. wort mit bewahrtem $n$ und suffixbetonung; die etymologie germ. pegno- = gr. $\tau \varepsilon^{\prime} x \nu o v$ habe ich in meinem et. wb. auf grund ganz anderer erwägungen bereits als zweifelhaft bezeichnen müssen. Auch got. $a b n$ 'jahr' setzt wurzelbetonung voraus.

Daher nehme ich betonung des $n$-suffixes als vorbedingung der germ. geminaten an mit Sievers Beitr. V, 149 anm. Es wären also worte wie ags. tâcen, fâcen, beacen urgerm. als wurzelbetont anzusetzen und die bewahrung ihres $n$ hätte nichts auffälliges. Die vermutung, dass $n$ in de: geminata untergegangen sei, beruht bekanntlich an der öfter erwähnten etymologie des germ. lokko- (lukko-) 'Locke' nach litt. lugna 'gebogen'; und in diesem beispiele spricht der wurzelvocal fur suffixbetonung, allerdings nicht unbedingt; doch ist das litt. wort und somit auch das germ. ein altes partizip mit dem stets betonten suffix idg. no.

Was die theorie des in der gemination untergegangenen $n$ zur gewissheit macht, ist die oben unter III B behandelte erscheinung wonach geminata in schwach flektierenden nominalståmmen besouders häufig auftritt. Wenn neben ahd. chnoto (chnodo) das ags. cnotta swm. steht, so lässt sich unschwer erkennen, dass das ags. wort das $a$ der schw. declination vom nominativ * cnoða (acc. * cnoðan) bezogen hat, da germ. knudnin der schwächsten stammform der schw. declination (got. aúhsnê, abnê) zu einem cons. stamm knutt- hätte fuhren mússen. Dem eigentlichen verhältnis steht ae. smocc gegen ahd. smoccho noch näher; idg. * smukin- musste * smugin-, ilg. smukn'- musste $s m u ̈ \bar{g}^{\prime}=$ smukk- ergeben: bei dem aussterben der ersten form trat die zweite in die a-declination (ae. smocc, an. smokkr), doch lezog die ahd. form noch vorhistorisch das o des nominativs und damit das prinzip der schw. declination von der form *smazim-. Auf iilnnliche weise verhalten sich an. bokki (ae. bucca) : ahd. bricch-; ahd. roccho : an. roklir (s. 165).

verartige contaminationen sind im bereich der germ. declinationen nicht selten. So ist der übertritt der $n$-stämme in no-stämme gut bezeugt durch an. vatn, nafn gegenüber dem 
got. watn-, namn- (nom. walò, namô); und ähnlich ist das verhältnis von an. geimi zu as. geban 'mcr', falls meine erklärung, Kuhns zs.26,87 das richtige trifft (stamnform gaimen-, gimeno:gimno-); und für ahd. raban, ramm (für ramn, rabn) gegem ahd. hrabo steht ursprung aus (hrahono- :) hrabno- gegen hrabondurchaus fest (vgl. ac. hrafn, an. hrafn wie an. nafn, watn). Auch an. gall n. 'galle' verhält sich zu einem vorauszusetzenden got. *galô schw. n. 'ff. gr. $\chi o \lambda \eta_{\text {) }}$ genau wie nafn zu namô, nur lass die genesis de $l l$ auf urgerm. ursprung des 0 -stammes weist. Dem gegec..oer kann ahd. galla f., ae. galla m. (got. * gallô neutr.) nur auf contamination des vorausgesetzten got. galò $(n)$ mit * gall- (für *galn-) beruhen ${ }^{1}$ ). Der alte neutrale osstamm ahd. sahar hatte urspringlich eine nebeuform * sahs wie neben ahd. ahir das got. ahs, an. ax 'ähre' besteht: auf einer contamination beider formen beruht ahd. sahsar. Aber auch ohne weitere belege ${ }^{2}$ ) ist die vorgebrachte erklärung der $n$ stämme wahrscheinlich, wonach aus *drupan- ein druppooder ein contaminiertes druppon- resultieren konnte.

Wie das erklärungsprinzip der eben behandelten nomina zuerst richtig von Osthoff beitr. 8, 299 anm. erkannt wurde, so verdanken wir ihm auch die lösung des problems der einfachen intensivbildung, für welche ich oben s. 163 material zusammengetragen habe. $\mathrm{Er}$ hält mit recht das ursprünglich bloss praesensbildende suffix $n a-(n u-)$ für den ausgangspunkt des bildungsprinzips aller primären intensiva des germ. Wenn nun dies praesenssuffix ausserhalb des germ. nie in ähnlicher funktion auftritt, so zeigen doch analoga, dass die ausbildung einer suffixbedeutung wol eine: einzelnen sprache zugeschrieben werden kann; hat doch wie Prof. Osthoff erinnert das alte praesenssuffix ske- im lat. inchoativbedeutung angenommen.

Der richtigkeit der von diesem gelehrten vorgeschlagenen

1) Für das westgerm. ist nach Osthoffs richtiger bemerkung Beitr. VIII, 300 anm. der gen. sing. ein bequemer ausgangspunkt gewesen, mag man nun westgerm. mann-es, naht-is mit wolfe-s oder * fòt-ós $\left(\pi \circ \delta-\delta_{\varsigma}\right)$ mit ae. genetiven wie pa-s, hwoe-s, rodera-s : roderaes, heofena-s : heofenoes vergleichen.

$\left.{ }^{2}\right)$ Ich erinnere noch an got. kinnus, angnus für * kinus, * angus QF. 32, 46; Anz. 6, 199. Dahin gehört got. mannus für *manus und wohl auch plaqus für * plakus, hnasqus fïr *hnaskus. 
theoric, aus den praesentischen $n$-suffixen die gemination der intensiva herzuleiten, tut es keinen abbruch, dass nur wenige der vorgcführten intensiva praesentia mit $n$-suffix ausserbalb

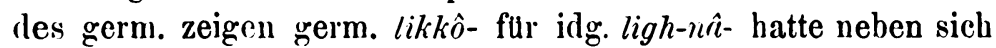
ein idg. ligh-nu- nach gr. $\lambda \iota \chi v \varepsilon \varepsilon^{\prime} w$. Das aus ahd. stëcchal 'steil' verschlossene stikkô- 'steigen' darf an ksl. stignati 'eilcn' und skr. stighnoti 'er schreitet' angeschlossen werden. Aber man ubersehe nicht, dass die meisten ir'‘nriva $\mathbf{z a}$ specifisch germ. wurzeln gehören. Bedenkt man :dass im Veda viele wurzeln mehrere präsensstämme neben érnander bilden, so scheint es unbedenklich ahd. zocchôn (: tiohan) auf idg. duknâmi (: deukô) zuriickzuführen.

Noch eine dritte erscheinung findet hier ihre erklärung. Wir haben oben mehrfach die tatsache beobachtet, dass neben geminata im selben wort einfache consonanz vorkommt; und s. 167 anm. wurde an die entsprechende erscheinung in got. ala-: alla-erinnert. Zweifelsohne ist alla- als alno- zu fassen und für ala- statt eines no- einfaches $o$-suffix anzunehmen. So dürften wir fur bakki- neben baki- (s. 167) suffix ni- annehmen und ähnlich abd. scopf : scof, chropf : chrof, chopf : chof verstehen. Also auch dies spricht zu gunsten von $n$ als letztem grunde aller gemination.

B. Wenn nun germ. $1 t$ aus $t n$ entsprungen ist, wie verhält es sich mit dem in 'I idg. dentalgeminaten' behandelten idg. $₫$ ? Mussten beide nicht einmal zusammenfallen? Thatsächlich sind beide im germ. durchaus verschieden (Möller, Beitr. 7, 469), und dies giebt uns momente zur genaueren fixierung der gemination in die hand.

Man kann also nur vorgerm. $"(t h t)$ für das jüngere $s s$ annehmen und von diesen vorgerm. $\|(t p t)$ muss also das $t$ von ae. cnotta 'knoten' (= ahd. chnodo, got. *knupa) immer unterschieden gewesen sein, weil dies nie zu ss wurde; d. b. um jene periode der lautverschiebung kann das germ. $\|$ (fur idg. (n) noch nicht bestanden haben. Der vorige abschnitt zeigte nun, dass ler idg. accent ein wesentlicher faktor in der genesis der geminaten ist, weil suffigiertes $n$ bei wurzelbetonung sich dem vorhergehenden verschlusslaut nicht angleicht. Demnach ist ags. cnotta nicht aus vorgerm. * gruttó- entstanden, 
weil dies zu * knusso hätte fibren müssen; viclmehr muss ags. crotta wie ahd. chnoto auf idg. gnutn'- (nom. gnutến) beruben, und aus dem durch die regel der lautverschiebung daraus entstandenen $\delta n(* k n u \delta n-)$ muss durch eine neue verschiebung tt geworden sein.

Wir kommen somit zur prufung des von Paul Beitr. VII, 133 aufgestellten gesetzes: „zwischen der urgerm. und der hd. lautverschiebung liegt ausser der durch Verner aufgeklärten noch eine weitere verschiebung, durch welche verschlusslenis zu verschlussfortis wird." Jedenfalls lässt sich die chronologie weit bestimmter fixieren auf grund der obigen erörterungen, welche ich gleich fortsetzen werde: ich glaube, die genesis der geminaten liegt zwischen Verners gesetz und der accentverschiebung, fällt also in die urgerm. zeit. 1)

Unsere geminatentheorie setzt die wirkung eines teiles der lautverschiebungsregel voraus: es werden die idg. tenues gleich behandelt mit den mediae aspiratae; daraus folgt, dass das gesetz uber die verschiebung der vortonigen tenues (resp. tonlosen spiranten nach Verner) zu tönenden spiranten bereits gewirkt hatte. Idg. gnutn- war durch knupn- zu *knuðn' geworden und $\partial n$ (resp. $d n$ ) war zu $t t$ geworden, weil aus idg.

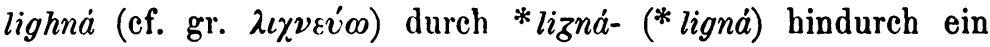
urgerm. *likkô- entsteht. Die beispiele für die gleiche behandlung der weichen aspiraten verdienen hier zugezogen zu werden. Ae. doppa 'mergus' gehört mit got. diups wahrscheinlich zu einer wz. dhubh, auf welche lit. dubus 'tief, hol' hinzuweisen scheint. - Ahd. stëcchal 'steil' beruht wie die nebenform steigal zeigt auf der idg. wz. stigh 'ascendere' (steigen) und setzt einen praesensstamm germ. stikkô- voraus, der sich mit ksl. stignati seiner bildung nach decken wurde wie das eben behandelte likkó- zu gr. $\lambda \iota \chi v-\varepsilon \dot{v} \omega .{ }^{2}$ )

1) Man könnte hier erwarten, dass dem skr. budhna 'boden' ein germ. *botto- entspräche; die dafür erscheinendeu ae. botm (: ahd. bodam $=$ setl $:$ sedal $=$ weolma $:$ widumo Kz. $26,98 \mathrm{f}$.) beruht auf mischung dieses botto- mit einem dem gr. $\pi v \vartheta \mu \eta \dot{v}$ entsprechenden bodmo-n-.

$\left.{ }^{2}\right)$ Pauls terminus ad quem ist zu weit gegriffen. Denn wie der verfolg der untersuchung ergeben wird muss vor der hd. verschiebung (noch urgerm.) ein weiteres gesetz gewirkt haben, wonach lange consonanz nach langer silbe gekïrzt wird. Ahd. eihhôn 'vinäicare' war vor 
Weit zahlreicher sind die fälle mit idg. tenuis im wurzelauslaut. Ahd. smoccho, an. smokkr beruben (grdf. * smuggnofür * snuggo-, *smugno') auf idg. wz. sm $\breve{u k}$ 'schmiegen' (lit. smukti 'gleiten', ksl. smucati 'kriechen', vgl. M. Unt. 4, 52). Fur ags. pryccan 'drücken' ergibt sich ein germ. wz. prûh, prùz aus ahd. drûh 'fessel', an. prúga 'dricken', also idg. wz. truk. Ahd. zocchôn neben ziohan zu germ. wz. tuh, vorgerm. duk. Ahd. * snizzôn (cf. s. 163) neben snidan, wz. snip, idg. snit. - Mhd. bücken (got. *bukkjan) aus wz. idg. buk (s. Beitr. 8, 2i8). - Ae. hoppian 'hlipfen' zu ksl. kypěti.

Bei einzelnen der oben vorgeführten beispiele lässt sich die vorstufe der geminaten nicht genauer fixieren, als dass idg. tenuis oder media aspirata zu grunde liegt. Vgl. ags. hôd: hoett 'hut'. - Ahd. flucchi 'flugge' neben fliogan, wozu auch ahd. floccho. - Ahd. zicchin neben ziga 'ziege'; ahd. chizzi neben an. $k i$.

C. Versuchen wir es die neu gewonnenen punkte in die chronologie der germ. verschiebung einzufugen, so bekommen wir folgende stadien in der entwicklung der urgerm. lautform aus der idg. lautform.

Neben den idg. verschlusslauten erbte das germ. keine geminaten ausser $t t$, das aber vorhistorisch vielleicht schon tbt war. Einen zuwachs erbielten diese geminaten vor der lautverschiebung nicht. Der erste schritt in der abweichung des dialekts von der ursprache war die ausbildung der spiranten fuir die aspiranten: eine grössere anzahl idg. dialekte teilten dieson wandel. Der erste akt der germ. lautverschiebung mochte die tenuesverschiebung sein, wodurch die fruber entstandenen spiranten an zahl gewannen. Der zweite akt war das Vernersche erweichungsgesetz, das alle vorhandenen liarten spiranten traf, einerlei ob sie alten harten aspiraten oder alten

der hd. verschiebung *aikôn; dies steht wegen eigan 'haben' für *aikkìn aus *aigg $\hat{o}^{\prime}-{ }^{*}$ aign $\hat{a}^{\prime}-$. Vielleicht ergibt sich noch ein weiterer punkt zur bestimmung der chronologie aus Sievers' gesetz vom ausfall des $\bar{\delta}$ vor $w$ : denn got. siuns für signni- war wol bereits entstanden, ehe $l_{\bar{\zeta} \not} n \hat{a}-z u$

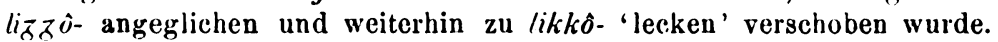
Leider fehlen weitere beispiele für un für gnn', so dass sich dies chronulogische moment nicht für sicher ausgeben lïsst. 
tenues entsprachen. Währond das alte $\|$ durch den ersten verschicbungsakt $\mathrm{zu} / \mathrm{h}$ geworden war wie $k l, \mu l \mathrm{zu} \chi l / l$, that nach vollzug des zweiten lautverschicbungsaktes, d. h. nach der wirkung des Verner'schen gesetzes, die angleichung der betonten $n$-suffixe an die vorausgehenden tönenden laute ein. Nachdem sich so das gebiet der tönenden verschlusslaute erweitert hat, tritt der letzte verschiebungsakt ein, wodurch alle medien, einfache wie geminierte zu verschlusslauten werden. ${ }^{1}$ )

Erst hier wo wie im zusammenhang der sonstigen lautverschiebungsverhältnisse das geminatenproblem betrachten, sind wir im stande eine innere chronologie der einzelnen erscheinungen $\mathrm{zu}$ gewinnen. Ich halte es für unnötig die annabme weiter zu verfolgen, dass die spiranten an stelle der

1) Soweit gehen die wege der gesetzlichen lautentwicklung, deren stadien einige charakteristische beispiele veranschaulichen mögen.

A. Vorgerm. periode.

(Entstehung der spiranten.)

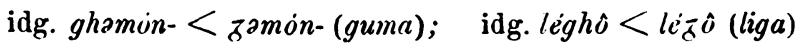

idg. dhures < Jures (an. dyrr); médhu <médu (meodu)

idg. bhid < bid (bîlan); nébhos < nébos (nëbal)

idg. rotho- <ro૭o- (ahd. rad); rêpho- <rêfo-(ahd.râvo).

B. Erster verschiebungsakt.

(T'enuisverschiebung.)

idg. bhrátôr < brátpôr (brôpar); patétr < fapér (fadar)

idg. woittha < woipta (waist); wittos < wiptos (guniss)

C. Zweiter verschiebungsakt.

(Verners gesetz.)

$$
\begin{gathered}
\text { fapér }<\text { futer } \quad \text { snusát }<\text { smuzát (ae. snoru). } \\
\text { D. Zwischenperiode. } \\
\text { (Geminirte medien.) }
\end{gathered}
$$

\begin{tabular}{|c|c|c|}
\hline 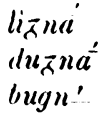 & $\begin{array}{l}<\text { ligga } \\
<\text { dugga } a^{\prime} \\
<\text { bugg! }\end{array}$ & $\begin{array}{l}\text { gnudn' } \\
\text { gnatin! } \\
\text { brgn }\end{array}$ \\
\hline
\end{tabular}

aber pizno-bleibt, ebenso rizno-.

\begin{tabular}{|c|c|c|c|}
\hline 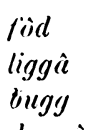 & $\begin{array}{l}<\text { föt } \\
<\text { likkì (liccônn); } \\
<\text { bukk (bock); }\end{array}$ & $\begin{array}{l}d e h \mathrm{n} \\
g n \tilde{u} d d \\
\operatorname{trgg}\end{array}$ & $\begin{array}{l}<\text { lichn (lchun) } \\
<\text { knï̈l (cnotta) } \\
<\text { brukk (broccho) }\end{array}$ \\
\hline $\operatorname{lig} g i$ & (zocchón) & gnabb & cnapp. \\
\hline
\end{tabular}

E. Dritter verschiebungsakt. (Medien werden tenues.) 
alten aspiraten zu den fribhesten errungensehaften der werm. lautlehre gehört: P'aul hat bereits Beitr. I, 199 dic möglichkeit crwiesen, „die verwandlung der aspiraten vor die andern verschicbungsakte zu stellen“. Dass die medienverschiebung der jüngste prozess in der eigentlichen verschiebung ist, ist auch bereits erkannt: nicht bloss das germ. Krēko- aus Graecus beweist dies; wären die tenues vor dem wirken des Vernerschen gesetzes entstanden, so dürfte man erwarten, dass dasselbe erweichungsgesetz, welches die tonlosen spiranten $z u$ tönenden macht, auch die tonlosen verschlusslaute $\mathrm{zu}$ tönenden gemacht hïtte; und man whide abwechselnd, je nach der accentstellung, tenuis oder media im germ. als vertreter der idg. mediae finden. Aber diese chronologie der verschiebungsakte ist längst bewiesen. Was sich hier als neues resultat dem anerkannten zufügen lässt, ist die genesis und die verschiebungsperiode der geminaten. Dass diese nach der wirkung des Vernerschen gesetzes entstanden sind, hat Paul zuerst erkannt. Wenn nun die jungste periode der verschiebung die genesis von (uberhaupt nicht mehr vorhandenen, erst neuen) tenues aus medien ist, soll man für die entstehung der geminierten tenues (likkôn, tukkôn, knuttan-, brukkon-, bukkon-) wider einen neuen späteren verschiebungsakt annehmen? Es liegt kein zwingender grund vor; denn zweifelsohne sind die doppelten tenues zunächst aus den weichen verschlusslauten entstanden (ligg $\bar{a}, d u g g \bar{a}$, gnuddon). Die einzige neue lautregel, die wir fur meine chronologie der gemiuaten nötig haben, ist der ubergang der langen

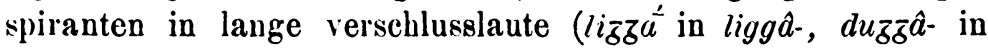
dugga, gnuððon- in gnuddon-. Dafur bieten sich die schönsten parallelen am späteren westgerm. gesetz der consonantendehnung vor jod (lizzian < liggjan, habtjan < habbjan u. 8. w.). Und was dic assimilierung anlangt, so muss die regel nun lauten: die weichen spiranten und verschlusslaute gehen bei suffixbetonung mit $n$ als suffixanlaut angleichungen ein. Einerseits wurde i.lg. bhugn! $<$ tugg $^{\prime}$, bhrgná- $<$ truggō; andererseits lizná $<$

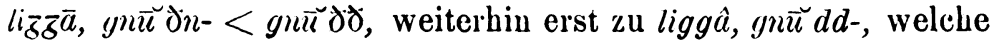
crst der letzte urgerm. verschiebungsakt zu likk $\hat{a}-$, knult- macht.

Wenden wir nun einen blick auf Pauls erklärung der probleme durch ein neues verschiebungsgesetz, so hat die erirterung gezeigt, dass seine annahme unstatthaft ist; es liegt 
kein grund ror zu einem neuen verschicbungsakt. Vielmehr fällt dies verschiebungsgesetz der geminierten medien genau zusammen mit dem letzten alst der germ. lautverschiebung, durch welchen einfache medien tonlos werden. Also nicht zwischen dic hd. und die urgerm. lautverschiebung, wie Paul wollte, fällt die genesis unserer germ. $k k t t p p$ als vertreter von idg. $k n g h g n$, $t n d h n d n, p n b h n b n$. Wir werden in der folge noch weitere momente aufdecken, welche die weit fruhere entstehung der tenuesgeminaten zwischen den beiden letzten akten der germ. lautverschiebung befürworten.

V. Geminierte medien und harte spiranten.

Es war das wesentliche resultat unserer erörterungen, dass das neue von Paul erkannte verschiebungsgesetz die aus $k n$ $g n p n$ und $g h n d h n$ bhn entstandenen $g g d d b b$ mit den durch blosse angleichung aus vorgerm. $g n, d n, b n=$ germ. $k n$ tn $p n$ entstandenen $k k t t p p$ gleich machte. Daraus ergibt sich dass germ. $g g, d d, b b$ nicht möglich sind, weil sie zu $k k t t p p$ werden mussten. Nach dem material des zweiten abschnittes gehören lange mediae auf dem germ. gebiet nicht zu den seltenheiten, wenn sie auch zufällig dem got. fehlen. Wie sind diese geminaten entstanden zu denken?

Dass neben ahd. chnabo eine form knapp- (aus knabn-) denkbar ist, ergibt sich aus dem bisherigen. Diese doppelformen fuhrten durch association zu zwei neuen formenpaaren: man bildete zu knato eine neue geminationsform knabba oder zu der geminierten form knapp- im anschluss aus knato eine form mit einfacher consonanz knapa: jenes ist das mhd. knappe, dies das ags. cnapa. Und nach änlichem prinzip erklären sich manche der s. $161 \mathrm{f}$. aufgefuhrten geminaten. Fllr ahd. roggo, rocko 'roggen' muss * ruggan- an stelle von * rukkan- (rukk-) im anschluss an eine stammform rugan- (resp. die nelonform rügi- Kuhns zs. 26,101) vorausgesetzt werden : rughon:rughn wurden zu ruzan- : rukk-; dafür tritt migan-: ruggan- ein. Genau wie knabe: knappe verhält sich mhd. rabe: rappe und so lässt sich auch begreifen, warum neben frocca 'frosch' im ae. cin frocga bestand: die urgerm. stammformen miissen *frugan-: * frukkgewesen sein, woraus beide ae. formen sich erklären lassen. 
Und das gleiche prinzip lässt sich auch verwerten für andere schw. masculina ohne nebenformen wie ae. crabla (\%u ahd. chrebiz), ëbba 'ebbe', ahd. svaggo - wacke 'stein', trappo 'traubenkamm', re. dorga 'bund', ae. budda 'käfer'; an. koddi 'tasche', ae. sceacga (s. 158).

Die schw. feminina miissen ähnlich erklärt werden. Zwar kommt innerhalb des germ. neben der starken resp. mittleren stammform ôn- keine schwache stufe mit blossem $n$ als suffix vor, aber sie muss nach den alten regeln jedesfalls vorauszusgesetzt werden; gelegentlich mag auch nebeu einem schw. fem. ein gleichbedeutendes schw. masc. oder neutr. gestanden haben. Mhd. lappe m. f. ist im ahd. zufällig nur als lappa f. bezeugt; vgl. das angls. masc. lappa 'zipfel' : ae. lapp-a weist auf germ. lapp-aus *labn-: * latôn-, wozu das deutsche dann ein *labbôn- schuf.

Für die s. 157 f. verzeichneten fälle von harten spiranten ist gleiche entstehung der geminaten aus inneren gründen wahrscheinlich; aber das material führt nicht selbst notwendig auf die gleiche erklärung. Da die beispiele fast nur dem westgerm. entnommen sind, wären noch allerlei andere möglichkeiten in betracht zu ziehen wie $\mathrm{z}$. b. die westgerm. consonantendehnung; könnte so ae. seohhe auf * sihwôn- beruhen, geneahhe auf * ganíhwô, teohhian auf * lihwôn? aber der dehnende einfluss des $w$ ist sehr beschränkt (ahd. quëcch-, nacchut aus qikkwo-, * nakkwed sind wol die einzigen sicheren beispiele). In folgenden ae. masculinen der schw. declination lässt sich das obige erklärungsprinzip anwenden: reohha, snoffa, pohha; vgl. noch mhd. schroffe s. 159 sowie das fem. ahd. laffa 'hand'.

Hier kōnnte der wechsel ae. lcetla (Stratmann) : * lackba (ne. lath) masc. erklärt werden; das fem. ahd. latta weist mit jenen auf vorgerm. látn: latôn', was auf labôn-: latt-führen musste; die letztere form ergab unter anlehnung an die flexion der ersteren cin lattôn-, die erstere unter einfluss des geminatenprinzips die form lappôn- = ahd. latta, me. labpe. So wäre denn auch neben ae. pipa 'mark' ein oben s. 160 vorausgesetztes * pippa wol denkbar; nach s. 160 neben ae. clipe 'klette' für alıd. chlëlto ein klippô. 


\section{Erschoinungen nach langer silbe.}

Ein besonderes interesse beanspruchen die geminationserscheinungen nach langer silbe. Leider ist das hd. der einvige dialekt, der für die seltenen erscheinungen als zeuge auftritt. Denn während die iibrigen westgerm. dialekte bei der jod-gemination langsilbige stämme unberührt lassen, führt allein das vorbistorische ahd., wie Paul Beitr. VII, 120 erkannt hat, die strenge regel nicht bloss durch, sondern bewahrt die reflexe dieser regel auch nach der lautverschiebung bis in die neuesten oberd. dialekte: während got. grôtjan, hraitja-, witjaim sonstigen westgerm. keine gemination zeigen, verschiebt das ahd. die regulär entstandenen grôttian, hwaittia-, wîttia- zu mbd. grüelzen, weitze, witze (slav. vice Scherer Anz. III, 64). Das gleiche musste auch von jeder sonst vorhandenen geminata gelten; d. h. nach langer silbe musste das hd. der treue bewahrer auch der urgerm. gemination sein, wo die sonstigen westgerm. dialekte keine spur derselben bewahren.

In der tat trifft dies zu. Nur das hd. bewabrt auch nach langer silbe spuren von germ. $g g, b b$, dd als $k t p$ resp. von $k k p p$ tt als $k \chi$ pf $t z$. Zunächst kommen wieder einige schw. nomina in betracht. Ahd. hâko, nhd. (els. schweiz.) hôken berubt auf *hêggo (Paul a.a.o.); ahd. chrîko 'haken' auf *krêggo; ahd. chrâpfo auf krêppo; ahd. gislâpfa 'nupta' (Marc. Cap. ef. Graff VI, 802; Weinhold al. Gr. s. 123) auf gaslêppòn- (cf. auch

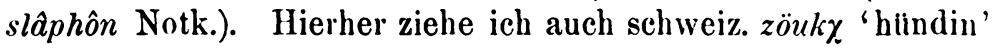
aus taukkiô- neben tauhô (= ahd. zôha, wol nicht zŏha wegen der schweiz. form ${ }^{1}$ )) Winteler s. 61 (die bildung von zöuk $\chi$

1) Die lïnge ist öfters schon z. b. Möller $K z .24,429$ angenommen; der hinweis auf die schweiz. form dïrfte die frage endgiiltig erledigen; natürlich ist die annahme von ablaut $u(\breve{o}): a u$ trot $z d e m$ nicht ausgeschlossen, wenn man einmal skeptisch sein will. Auch mhd. (rheinfriink. ndrh.) züpe, nhd. zaupe (bei Weigand) 'hündin' gehört wol zu den fïllen von urgerm. $b b$. Hierher auch schweiz. znupe 'knäuel', das nach Winteler p. 59 auf germ. "knùbba weist und zu knüpfen (mhd. knübel, me. ndl. knobbel) gehört? Wintelers beobachtungen s. 59 ff. liefern weiteres material für die erscheinungen nach langer silbe; uur lassen sich die betr. worte historisch nie weit zurïckverfolgen, weshalb mehrfach die annahme westgerm. consonantendehnung der grund eines oberdeutschen $k(t \mu)$ sein kann. Hierber gehört aus dem schwäb. naupe : nuppe 'grille' wit $p=b b ;$ s. Lexer DWb. - Alıd. hâppa vgl. s. 16i2. 
neben zôha deckt sich annähernd mit der Paul aufgedeckten beziebung ricke : rêh Beitr. VII, 133). Auf mhd. leuken, loukenen 'leugnen' (md.) aus lauklijan, lauklianôn hat Paul ebenda aufmerksam gemacht, ebenso auf mhl. (mol.) nêken 'nahen' aus nâkkian. Mhd. tâpe (els. dòpen) nı. 'pfote' aus * dêbban-, mhd. snâke (snûcke, els. snìke) mf. 'schnake' aus snêggun-, schuope, ahd. scuoppa 'schuppe' (zu skaban), mbd. snûpfe neben snupfe (ae. snoffa), nhd. schnautze aus snktta- vgl. ndl. snuil (me. snont), nhd. kautz u. a. zeigen umlautslose gemination.

Das ahd. rûppa und scâpâri, mhd. schâpcere. ( $p$ aus bb) 'vellus, schaffell', eine auffällige bildung zu ahd. scâf (germ. skêpo-) legt die rermutung nahe, das öfters, in Genes. Exod. allein achtmal bezeugte $b$ von ae. tîber 'opfer' als verkur\%ung fur $b b$ zu betrachten: an stelle der weichen spirans war in der gemination schon urgerm. die media eingetreten, und wenn nach dem oben angedeuteten gesetz auf den ausserhd. gebieten auch vereinfachung der gemination eintrat, so musste eben einfache media (zunächst noch nicht die weiche spirans) eintreten. So ist auch das dem ahd. daucgal entsprechende ae. deacgol mit seinem $c g$ nicht anders zu beurteilen als etwa cynincg : $c g$ meint die einfache media. Dem ahd. rûppa 'raupe', nhd. graupe entspricht ndd. rube, grube mit $b=b b$ Corresp.Bl. f. ndd. Spr. V, 94.

Ob auch nach consonanten $g g, d d, b b$ im vorhd. möglich war? Das auftreten von $p$ und $k$ in streng oberd. dialekten könnte dafür sprechen. Winteler s. $58 \mathrm{ff}$. fluhrt beispiele für $p$ nach $m r l$, für $k$ nach $n$ an; der Kerenzer und 'Toggenburger mundart gemäss wäre z. b. für zinke ein * tinggo (resp. tinyjo), für lunke 'lunge' ein *lunggo u. s. w. vorauszusetzen. Aber ich finde kein beispiel, in welchem sich eine jung bezeugte form mit notwendigkeit und historischer sicherheit auf eine urgerm. geminata zurückführen liesse. Fur die ältere zeit ist wülpe, ahd. wulpa Beitr. VII, 133 ein beispiel fïr diese erscheinung nach dem westgerm. prinzip der gemination, ebenso rinke 'spange' (aus hringjô?? vgl. Winteler s. 62, Paul Beitr. VII, 133).

Das erklärungsprinzip der geminaten von *hêggo (hâko), * krêppo (cbrâpfo), *slêppô (gislapfo), * dêbbo (* tâpo), * snêggo (*suâko) - lauter schwach flecticrende nomina - muss naturlich mit dem bisher behandelten eins sein. Wir werden dem- 
nach in diesen beispielen contaminationen aus formen wie *hêzon- mit der gesetzlichen *hêkk-zu erkennen haben. Auch finden sich hier genau dieselben zwillingsformen wie oben; *hêkk- wurde ausserhalb des bd. zu hêk- (ndl. haek-), und contamination mit hêzon- konnte zu hêkon- (an. haki, ae. haca haben aber jedenfalls kurzes a) führen, woneben noch die formen ae. hôc, ne. hook, ndl. hoek als ablautsform sich einstellen: hêzón- (*hổón-) : hêk (hôk) : hêkon- (*hôkon-) = knato:* knappo: knapo. Und wie troffo: tropfo, so verhalten sich chrâfo : chrâpfo $\left.(p: p p)^{1}\right)$ und chrâgo: chrâko $(g: g g)$, das noch die nebenform an. krókr, krákr 'haken' hat.

\section{Indogerm. wechsel von tenuis und media.}

Osthoff hat jüngst in den Morph.-Unt. IV, 328 auf eine vorhistorische erscheinung hingewiesen, die in einem noch nicht genau zu formulierenden vorhistorischen wechsel von tenuis und media im wurzelauslaut besteht; nach Osthoffs vermutung ist dabei die nachbarschaft von nasalen erforderlich. Von den beweisenden momenten ausserhalb des germ. führe ich nur einiges an.

Skr. rgmin 'singend, jubelnd' zu rc 'lied, besingen' (arká 'sänger'). - vagnú 'getōse' zu wz. vac 'reden'. - cagmá 'vermögend' zu wz. cak 'vermögen' (cakrá 'stark'). - huj 'nachkommenschaft' zu tuc, toka. - Wz. tuj: tuc 'reichlich spenden'. - pajrí 'fest' zu idg. wz. pak'. - Aus dem gr. gehören hierher wz. $\varphi v \gamma$ aus idg. wz. bhuk (Beitr. VIII, 278). -

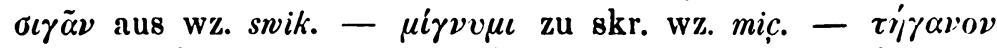

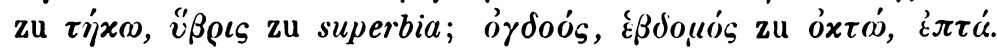
- Aus dem lat. kommt etwa mendax zu mentiri, dignus zu

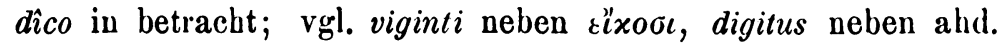
zêha; vgl. Curtius Grdz. s. 522.

Er gibt durchaus keine specialgesetze, nach denen der hier bezeugte wechsel von tenuis und media zu erklären wäre: so kennt die altind. grammatik für die medien von çagmá,

1) Daneben erweist das DWb. auch krâpe als echt oberd.; vorauszusetıendes $k r e \hat{b} b a: k r e ̂ p p a=$ oberd. lappe : ae.lappa (s. 165) = oberd. hoppen : hopfen (s. 163) = oberd. schoppen : schopfen (s. 162). 
rgmin, vagmi u. s. w. keine regel. Wir haben es eben mit den letzten ausläufern einer altidg. regel zu tun, welche auch im germ. ihre spuren hinterlassen hat. Man hat schon fruher auf hierher gehörige erscheinungen geachtet und die scheinbare störung vor die germ. lautverschiebung zurückdatiert. Osthoff hat das alter der lautregel erkannt, und so lässt sich mit dem factor der erweichung jetzt sicherer operieren als früher. Wenn so im ältesten idg. nach einer vorhistorischen lautregel $k$ und $g$, $t$ und $d(p$ und $b$ ) im auslaut derselben wurzel auftreten können, so dürfen wir uns nicht wundern im germ. den parallelen wechsel von $h(\zeta)$ mit $k, b(\delta)$ mit $t, f(\zeta)$ mit $p$ zu finden. Ich sehe hier ab von den fällen der gemination, welche durch einc specifisch germ. lautregel zu erklären sind. Aber es können hier zahlreichere andere unklarheiten im germ. consonantismus beseitigt werden.

Das gemeingerm. taikno-, taikni- aus idg. dóigno-, dóignizu wz. germ. tih, idg. dik hat mit dem geminationsgesetz keine berthrung, weil daikko-, daikki- (resp. daiko-) zu erwarten wäre; wahrscheinlich war das wort urgerm. paroxytoniert; das ab). geleitete, schw. verb muss als oxytoniert fur das urgerm. vorausgesetzt werden (ae. tốcan aus doiknéjô oder doignéjô). Aehnlich verhält sich ae. fâcn (ahd. feihhan) aus fáikno- (vorhistorisch póigno-) zu got. faihô 'betrug', wz. pik; germ. wêpnoaus vorhist. wèbno- zu gr. ö $\pi$ גov.

Auch nacb nasalen zeigt sich im germ. ein consonantenwechsel ähnlich dem von lat. mendax: mentiri. Zu got. hinpan gebört ae. huntian, ne. to hunt; ac. steht veben cringan ein crincan, neben swongor ein swoncor; ähnlich verbält sich ahd. ringan zu renkî. Doch lassen alle diese beispiele eine andere beurteilung $\mathrm{zu}$, wie der folgende abschnitt zeigt, wo eine grössere anzahl von einschlägigen worten gegeben ist.

Aber auch sonst kommt germ. wechsel von $h(z): k, p(\delta)$ $: t$ und $f(t): p$ vor. Ahd. tougan 'heimlich' aus idg. wz. dhuk (: dhug) hat im ahd. ausser firtuhhilen 'verheinlichen' noch das particip firtohhan 'verborgen' (Graff V, 368) neben sich, das nicht auf einer grdf. dhuknó- oder dhugnó beruhen kann; tougan ist vorhistorisch dhoukóno-, tohhan ist dhugóno-. 1) Zu ae. drŷge

1) Nasaliert ist die wurzel dhuk (dhug) in ahd. tunchal 'dunkel'. Beiträge zur geschichte der deutschen sprache. IX. 
'trocken' aus dhrüki- gehört altsüchs. drokno und ahd. trocchano,

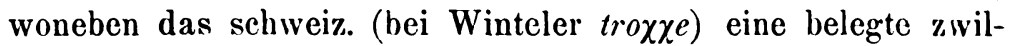
lingsform truhhano trohhano voraussetzt, die nur aus vorhistorischem dhrugono- erklärt werden kann; mhd. truchen adj. belegt Lexer. - Aehnlich wird das von Möller Kz. 24, 465 erwähnte ndd. fâken adv. 'oft' $\mathrm{zu}$ gr. $\pi v x v a$ ' 'oft' ( $v=0$ wie in $v v \varkappa \tau-$, ovv $\chi_{-}$) stimmen, und auch got. hatis kann so mit gr. xótos und hd. hader verwant sein.

\section{Scheinbaro ausnahmen der lautverschiebung.}

Solche störungen, denen eine altidg. lautregel zu grunde liegt, bietet das germ. noch in weit grösserem umfange als der vorige abschnitt andeutete. Nicht bloss in der umgebung vou nasalen findet sich ein wechsel ron germ. spiranten und tenues, denen idg. tenues und mediae $z u$ grunde liegen. Aber der mangel eines nasals kann ja secundär sein, indem das angleichungsgesetz ein vorhandenes $n$-suffix vernichtete. So könnte ahd. falzên, nhd. falzen (aus *faltai-) neben falban wol auf falddai- < fallai- beruhen und ein idg. pllnâ zur voraussetzung haben, obwol wach dem erweichungsgesetz des vorigen abschnittes die möglichkeit ciner idg. nebenform plday(resp. auch pldnâ-) nicht ausgeschlossen ist. Beispiele dieser art bietet das germ. zahlreich: bei ihnen ist also eine doppelte erklärung denkbar; aber keinenfalls darf man in ihnen erusthaft ausnahmen der germ. lautverschiebung erblicken. So wird got. pairkô- 'loch' gegen ae. phyrel (für '* pyrhel) 'löcherig', got. pairh 'durch' wol auf dem erweichungsgesetz beruhen (terk: (erg). Und hilpan 'helfen' könnte mit skr. klp 'ordneu, bereiten' ebenso verglichen werden; doch besteht die möglichkeit, dass das $p$ von hilpan auf idg. pn (klpna-) beruht. Hierher ziehe ich noch folgendes: ahd. stumpf 'verstummelt' neben gleichbed. stumbul, ahd. mhd. krumpf 'krumm' neben gleichbed. krumb (ae. crumb); ald. chlampfaròn 'klammern' neben mhd. klempern; mbd. klimpfen 'klimmen' neben gleichbed. ahd. chlimpan; ahd. wanchôn, wanchilòn neben lat. vacillare, skr. vañkri 'sich tunmelnd'; ae. wrincle 'runzel', ae. nrenc 'list', ahd. renchi 'drehung' zu ahd. ringan, ae. ureon (aus "riñhan, wz. idg. wrenk) 'drehen'; mhd. henken (nhd. schweiz. henkien) 
zu wz. hañh; ae. crincan, cringan 'fallen', wozu ahd. chrancholion, ae. cronc, ae. slincan 'schleichen' neben ahd. slingun; ae. swancor neben ahd. swangar (ahd. mhd. swenken); ae. sùcan neben sûgan 'saugen'. ')

Dass man in allen diesen fällen Osthoffs erweichungstheorie für das germ. wirksam sehen kann, lässt sich nicht läugnen. Ob man diese annahme als nötig erachtet, hïngt davon ab, wie man sich zu folgender erörterung stellt.

Osthoff hat bereits Beitr. VIII, 299 mitgeteilt, dass ich seiner ansicht beipflichte welche eine urgerm. verkiirzung von tt $p p k k$ nach langer silbe verlangt. So könnte germ. hwiı 'weiss' für hwîtto- gekürzt sein, und dies für hwidni- auf kwîlni- (skr. cuitna, cvilnyci zu evêta) hinweisen. Freilich sollte man nach unserm sechsten abschnitt wol hwitlo- auf hd. gebiet durch * witz, nicht durch $n \hat{\imath} z$ reflectiert erwarten (das von Weinhold baier. gr. s. 155 einmal aus Mon. Boica belegte weilz kommt gegen das durchgängige $z$ nicht in betracht). Ich nebme daher wegen des gemeingerm., durch das hd. vorausgesetzte einfachen $t$ urgerm. kürzung des hwitto- $<$ hwito- an. Das verhältnis zu den erscheinungen des sechsten abschnittes denke ich mir folgendermassen: Nachdem die geminierte tenuis durch das neue verschiebungsgesetz an umfang zugenommen hatte, trat zunächst nach langer silbe stets kürzung der doppelconsonanz ein: so denke ich mir neben hèzon- eine urgerm. nebenform hêk-(für hêkk-); neben krèpon-eine form krêp (für krêpp$=k r \hat{e} p n-)$. Denn nur so lässt sich hd. niz, ahd. eihhôn 'vindicare' (für aikô- $=$ aikkô- $=$ vorgerm. uiknâ-) denken, rgl. ac. lôcian für lòknai- = lokkki- gegen ahd. Iungin; ahd. wihhan aus germ. wiko- $=$ wikko- (gr. $\varepsilon i x(0)$. Der conson. stamm ae. nic 'flecken', als urgern. wort gefasst, beruht auf idy. wikn(zu lat. vicus, skr. viç²). Für an. knútr vermutete Ostboff Beitr.

1) Einzelne von diesen beispielen hat Osthoff in seinen aufsätzen Beitr. $S, 256$ unter dem gleichen gesichtspunkt besprochen, andere Möller Kz. 24, 441. 517; Beitr. 7,460 : vou beiden gelehrten sind noch weitere beispiele beigebracht, deren erklärung sich hier von selbst ergibt.

2) Aus den urkunden ergibt sich wic als fem. consonantischer stanm Kemble VI, 14s. 217; vgl. auch Hom. I, 402. II, 374. 382 sowie das Gloss. zu Blick. How. - Ae. dìc als fem. cons.-stamm folgt aus Cod. Dipl. V, 191. 193. 295. 344. 346. 376. 379. VI, 2- u.s. w. u.s. w. Es künnte

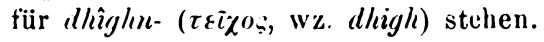


VIII, 299 anm. entstehung aus knutt- für knâtn- (: knŭtn- in ae. cnotta neben ahd. chnodo. chnoto). $\mathrm{Zu}$ ahd. blôz, ae. bleat für blautto- gehört an. blaupr, ahd. blôdi (got. blaupjan), wz. bhlaut-. Ob ahd. chnouf aus knaupo- fur knauppo- neben knopf auf einer wz. gnup beruht, lässt sich wegen alıd. chnubil 'knōchel' (s. 178: nicht entschciden. Neben ahd. huoh, ae. hôh 'spott' weist auf germ. $k k=k n$ (idg. $k n$ ) ae. hôcor (= hâknas).' So verhält sich auch an. fraukr zu ae. frocga. Gegenüber diesen zahlreichen formen, welche das verkürzungsgesetz befüworten, treten der zahl nach die wenigen beispiele für consonantendehnung bedeutend zurück. Ich erblicke in ihnen jüngere reflexe der sonst weit verbreiteten consonantendehnung nach kurzen vocalen: wie neben knato ein knabbo bestand, so schuf man zu hêzon- ein hêggon-, zu krêzon- ein krêggon-; und doppelformen wie dropon- : droppon- bildeten das muster für krêpan: krêppan-. Nach diesem prinzip lassen sich alle durch das ahd. vorausgesetzten geminaten nach langer silbe erklären, soweit nicht das gesetz der westgerm. consonantendehnung ihre ursache sein kanu. Und so können wir fur das urgerm. das von Möller und Osthoff empfohlene prinzip der urgerm. kürzung langer consonanten getrost gelten lassen, wonach hwitco- urgerm. zu hwîto-, sûkko- (ae. sûcan) zu sûko- wurden, germ. haupo- 'Lauf' für huuppo-=haubnó-=koupnó zu ksl. kupŭ steht.

IX. Consequenzen und resultate.

1. Die bisherigen erörterungen legen es mir nahe ein problem zur sprache zu bringen, das von der ältern grammatischen richtung mit eifer verhandelt wurde ohne einen abschluss gefunden zu haben: ich meine die genesis des inlautenden germ. $p$, welches in der gemination und nach natur- oder positiouslanger silbe durch die frluheren abschnitte begreiflich gemacht ist. In der tat fallen unter die aus dem vorigen sich ergebenden erklärungen die meisten germ. $p$ ohne dass die voraussetzung eines idg. $b$ nötig wäre. Freilich wird sich die existenz eines idg. $b$ weder für den an- noch für den inlaut läugnen lassen. Die ubereinstimmung von skr. pibâmi mit lat. bibo, ir. ibim (idg. pibômi zu Wz. pô), vou skr. ramb 'schlaff herabhängen' mit lat. 
labi 'gleiten', von ksl. slabŭ 'schlaff' mit gleichbedeutendem germ. slapo- sowie das auftreten von $b$ in vedischen worten wie bála 'kraft, stärke', sabar 'nektar' (dazu ae. sap, ahd. saf 'saft'?), rhî̌š 'schlund', bali 'spende'; bîja' 'same' (ich sehe dabei ganz ab von vedischen $\alpha \dot{\pi} \pi$. $\lambda \varepsilon \gamma$. wie brbukka, bêkanâta, bilma, bisa, bîrita, basta, basri, bâni u. s. w.), dies alles beweist dass auch die idg. grundsprache gewiss schon einige $b$ hatte; aber es war sicher im inlaut nicht häufiger als im anlaut. Das germ. mit seinem so unzäligemal im inlaut auftretenden $p$ wurde eine sonderbare unbegreifliche eigenart an den tag legen, wenn sich diese vielen $p$ nicht nach den fruheren gesetzen aus idg. $p n$ resp. bhn erklären liessen. So begreift sich denn das seltẹne $p$ im anlaut von echt germ. worten gegenuber dem häufigen $p$ im inlaut. Es bleiben freilich auch einige germ. $p$ (bes. nach kurzer tonsilbe) ubrig, fur welche jene erklärung nicht passt. Wenn ich die von Bechtel erkannte genesis von $p$ für $q$ aus idg. $g^{2}$ (Bezz.-Beitr. V, 169) hier ebenso aus dem spiele lasse wie die $p$ von sichern oder wahrscheinlichen fremdworten (s. mein etym. wb. s. hanf), so bleibt nur ein idg. $b$ als quell der ubrigen wenigen germ. $p$ zurtick. Aber fur einige derselben ergibt sich noch eine weitere möglichkeit. Wenn neben knopf (germ. knoppo-) und knauf (knaupo- für knauppo-) ein mhd. knübel 'knöchel' steht, so weist dies deutlich auf wz. idg. gnup resp. gnubh und dazu könnte ein gern. knopo- (ahd. chnof, nhd. knoff s. Dwb.) eigentlich nicht gedacht werden; wenn es nun aber doch vorhanden gewesen sein muss, so darf man aus den germ. knoppo-: knaupo- für das germ. sprachgeftuhl eine neue wurzel knop mit einfachem $p$ schliessen; auf ähnlichem prinzip, das auch den parallelen wechsel von knocke, knoche, knügel 'knöchel' erklärt, werden manche von den s. $165 \mathrm{ff}$. angeflihiten doppelformen einiges von ibrer schwierigkeit verlieren.

2. Ein weiterer schluss bleibt aus unseren erörterungen noch zu ziehen. Während $t n, d n$, $d h n$ im germ. nach s. $171 \mathrm{zu}$ jungem $t t$ wurden, haben wir kein beispiel kennen gelernt, in welchem dies nach vorherigem nasal eingetreten ist. Das oben angeführte einzige ae. huntian neben got. hinpan steht parallel dem lat. mendax neben mentiri. Aber jenes fehlen für $n t(=n t t)$ als reflex von idg. $n t n(n d n, n d h n)$ hat 
einen durchsichtigen grund: dicse lautcomplexe wuden vorhistorisch durch ausdrängung der verschlusslaute (implosiva) bequemer sprechbar gemacht. Ich habe bereits Beitr. VIII, 518 fiir die erscheinung einirc belege beigebracht: ahd. hunno ' centurio' für kntni- (älter kmtni-), ahd. sinnan 'grehen' zu sinf, 'weg' für senıno-; äbnlich verhält sich ahd. zinna 'zinne' zu mhd. zint, an. lindr 'zacke', ahd. zammin 'ringere' zu zand 'zahn' (grdf. idg. dentnâ-, dontnay-). lch vernute, dass germ. rinnan für rintno- steht; das germ. verbalabstrakt ae. ryne scheint zwar auf eine wz. ren zu weisen, kann aber eine nachbildung von bryne zu brinnan sein; das verbalabstrakt germ. runsi- 'lauf" (got. ahd. runs) muss mit suffix -li- grebildet sein und setzt daher eine grdf. rntli- nach s. 150 f. voraus; auf idg. wz. rent weist auch ae. ride 'bach'.

3. Als das hauptresultat unserer einzelnen untersuchungen darf ich zum schluss die sätze hinstellen, dass die im germ. so zahlreichen langen tenues - eine dem ilg. noch fremde consonantenform - keinem neuen verschiebungsgesetz ibr dasein verdanken. Sie beruhen auf dem gesetz, wonach auch einfache media tonlos gemacht wird. Ein neues moment fur die urgerm. lautgeschichte ist neben der längst erkannten assimiliruugsfähigkeit des suffigierten $n$ bei suffixbetonung (Sievers Beitr. V, 149) der ubergang der langen tönenden spiranten in lange tönende verschlusslaute $(z z<g g$, $\quad j t i<b b$, $\partial \delta<d d$ ), deren verschiclung also mit der behandlung kurzer medien durchaus gleichzeitig gewesen sein muss. Wenn nun anstatt der lautlich allein berechtigten langen tenues in historischer zcit auch lange mediac und spiranten erscheinen, so konnten diese auf nachbildung der grossen kategorien der geminierten tenues, welche oft mit harten oder weichen spiranten wechselten, zurtickgeführt werden. Eine weitere urgerm. lautregel verlangte die kürzung langer tenues nach langer silbe; wenn auch hiervon einige wenige ausnahmen begegnen, so können auch diese auf nachbildung beruhen.

STliASSBURG, 15. januar 1883.

F. KLUGE. 